\title{
Hematopoietic stem cells are acutely sensitive to Acd shelterin gene inactivation
}

\author{
Morgan Jones,, ${ }^{1,2,3}$ Gail Osawa,, Joshua A. Regal,,3,5 Daniel N. Weinberg, ${ }^{1}$ James Taggart, ${ }^{1}$ \\ Hande Kocak, ${ }^{6}$ Ann Friedman, ${ }^{1}$ David O. Ferguson, ${ }^{5}$ Catherine E. Keegan,4,6 and Ivan Maillard ${ }^{1,7,8}$ \\ ${ }^{1}$ Center for Stem Cell Biology, Life Sciences Institute, ${ }^{2}$ Graduate Program in Cellular and Molecular Biology, \\ ${ }^{3}$ Medical Scientist Training Program, ${ }^{4}$ Department of Pediatrics, ${ }^{5}$ Department of Pathology, \\ ${ }^{6}$ Department of Human Genetics, ${ }^{7}$ Division of Hematology-Oncology, Department of Medicine, and \\ ${ }^{8}$ Department of Cell and Developmental Biology, University of Michigan, Ann Arbor, Michigan, USA.
}

\begin{abstract}
The shelterin complex plays dual functions in telomere homeostasis by recruiting telomerase and preventing the activation of a DNA damage response at telomeric ends. Somatic stem cells require telomerase activity, as evidenced by progressive stem cell loss leading to bone marrow failure in hereditary dyskeratosis congenita. Recent work demonstrates that dyskeratosis congenita can also arise from mutations in specific shelterin genes, although little is known about shelterin functions in somatic stem cells. We found that mouse hematopoietic stem cells (HSCs) are acutely sensitive to inactivation of the shelterin gene Acd, encoding TPP1. Homozygosity for a hypomorphic acd allele preserved the emergence and expansion of fetal HSCs but led to profoundly defective function in transplantation assays. Upon complete Acd inactivation, HSCs expressed p53 target genes, underwent cell cycle arrest, and were severely depleted within days, leading to hematopoietic failure. TPP1 loss induced increased telomeric fusion events in bone marrow progenitors. However, unlike in epidermal stem cells, p53 deficiency did not rescue TPP1-deficient HSCs, indicating that shelterin dysfunction has unique effects in different stem cell populations. Because the consequences of telomere shortening are progressive and unsynchronized, acute loss of shelterin function represents an attractive alternative for studying telomere crisis in hematopoietic progenitors.
\end{abstract}

\section{Introduction}

Linear chromosomes are capped with telomeres to protect their ends from the loss of genetic material during strand replication. Disruptions in the stability of this molecular buffer have been linked to organ failure, aging, and cancer. Privileged compartments, including the germline and somatic stem cells, express the ribonucleoprotein telomerase to maintain telomere length during replicative stress $(1,2)$. When this activity is impaired, stem cell populations become depleted, leading to loss of tissue homeostasis (3). In addition, telomeres must be protected from the DNA damage response that would perceive telomeres as sites of DNA breaks, a function achieved by the shelterin complex. Together, the six shelterin proteins TRF1, TRF2, RAP1, TIN2, POT1, and TPP1 not only protect telomeres, but also recruit and regulate telomerase activity (4). Understanding the biological functions of these proteins is therefore critical to understanding telomere homeostasis and human diseases related to dysfunctional telomeres.

Studies using mouse embryonic fibroblasts (MEFs) and other culture systems showed that individual shelterin proteins have specific functions in suppressing the DNA damage response and in telomere regulation. POT1 binds the single-stranded telomeric overhang and prevents ataxia telangectasia and Rad3-related (ATR-related) kinase activation $(5,6)$. POT1 binding requires TPP1, the protein product of the ACD gene (also named TPP1, PTOP, PIP1, and TINT1) (6-8). In addition to being essential for POT1 recruitment, TPP1 recruits telomerase to the telomeric end and is required for telomere extension $(9,10)$. TRF1 and TRF2 bind the double-stranded portion of the telomere (11-13). TRF2

Conflict of interest: The authors have declared that no conflict of interest exists. Citation for this article: J Clin Invest. doi:10.1172/JCI67871. prevents ataxia telangectasia mutated (ATM) kinase from mistaking telomeric ends for sites of DNA breaks $(5,14)$. TINF2 stabilizes TRF1 and TRF2 at the telomere and binds to TPP1, linking the single-stranded and double-stranded binding portions of shelterin $(15,16)$. RAP1 interacts with TRF2 and prevents aberrant nonhomologous end joining from occurring at the telomere (17-19). In mice, studies of the shelterin complex are complicated by the duplication of the Pot1 gene into Pot1a and Pot1b (20). POT1A prevents ATR activation, while POT1B prevents excessive $5^{\prime}$ resection at the telomere and the consequent generation of excessive $3^{\prime}$ overhangs (20). In humans, a single POT1 protein accomplishes both of these functions (21). To obtain complete loss of POT 1 function in mice, either Pot $1 a / b$ double-deficient mice must be used, or Acd must be inactivated. In embryonic fibroblasts, both $A c d$ inactivation and Pot $1 a / b$ deletion caused rare telomeric fusion events and proliferative arrest, a phenotype that involved p53-driven expression of the cyclin-dependent kinase inhibitor $p 21(6-8,20)$.

Although embryonic fibroblasts have been a useful tool in understanding the molecular functions of the shelterin complex in cell culture systems, the physiological role of shelterin components in vivo remains poorly understood, especially in tissues maintained by somatic stem cells. Recent studies demonstrated that when combined with telomerase haploinsufficiency, Pot1b deficiency resulted in a gradual decline in tissue homeostasis similar to that observed in late-generation telomerase-deficient mice (22). These mice displayed skin hyperpigmentation and bone marrow failure reminiscent of those found in human patients with the telomere shortening syndrome dyskeratosis congenita (DKC). DKC has been linked to mutations in the telomerase component genes TERC and TERT, or in $D K C 1$, encoding the telomerase accessory protein dyskerin (23). 

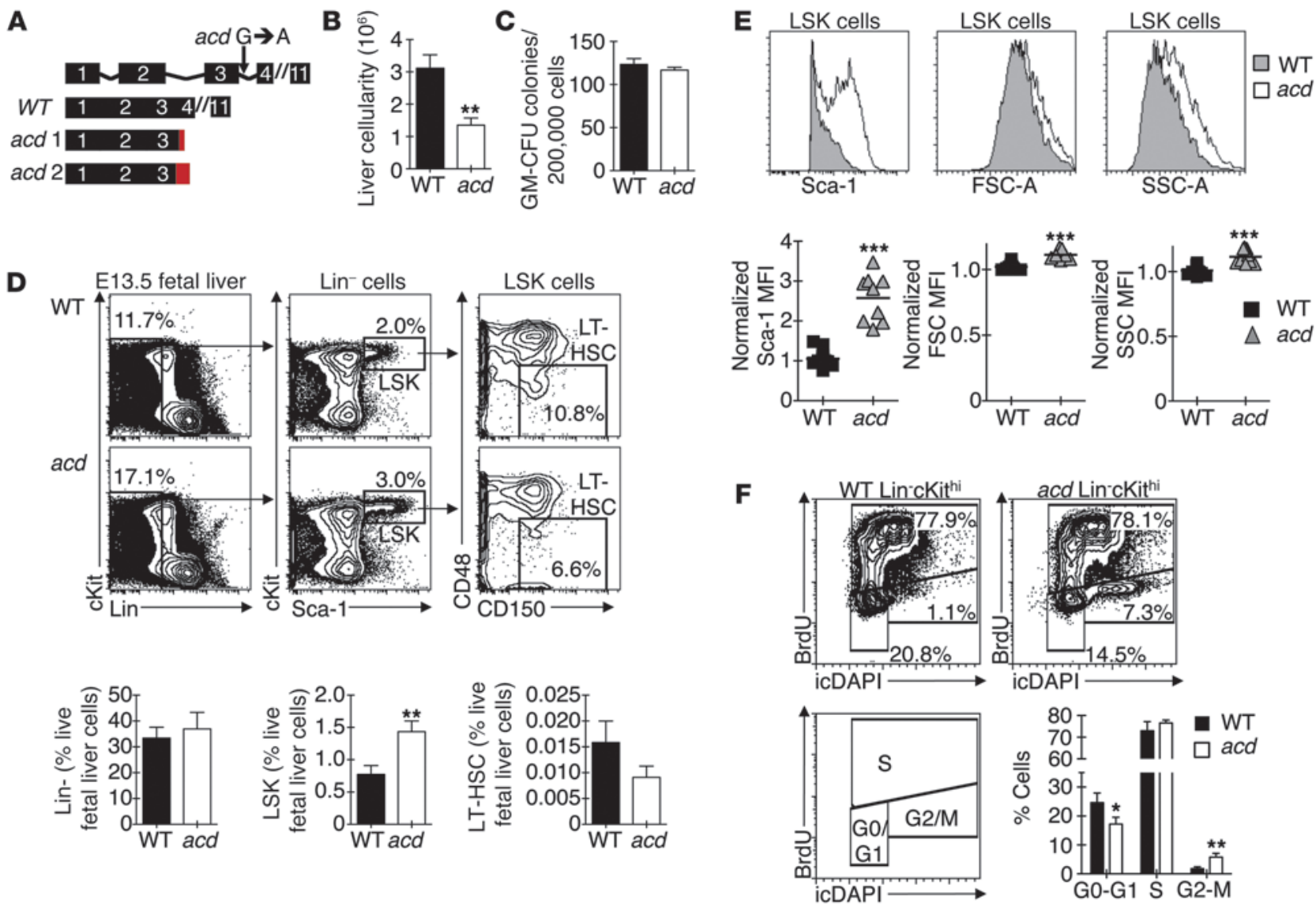

\section{Figure 1}

Reduced Acd expression results in phenotypic abnormalities and G2/M arrest in fetal hematopoietic progenitors. (A) Structure of the hypomorphic acd allele, a mutant Acd allele arising from an intron $3 \mathrm{G} \rightarrow \mathrm{A}$ transition and leading to aberrant splicing. acd homozygosity decreased the abundance of normal transcripts to approximately $2 \%$ of WT (33). (B) Fetal liver cellularity in E13.5 mice homozygous for the acd hypomorphic allele. (C) GM-CFU analysis demonstrating preserved GM progenitor activity in E13.5 acd fetal liver (representative of three experiments with triplicate colony formation assays; mean \pm SEM). (D) Preserved overall frequency of phenotypically defined long-term hematopoietic stem cells (LT-HSC), defined as CD150+CD48- Lin-Sca- 1 hicKithi (LSK) cells ( $n \geq 9$ mice per group from five independent experiments; mean \pm SEM). (E) Increased Sca-1 expression in acd LSK progenitors. acd LSK cells were also larger and more granular by forward (FSC-A) and side scatter (SSC-A) characteristics, respectively ( $n \geq 9$ mice per group from five independent experiments). Gray shading shows data from control littermates, and white shading represents acd progenitors. (F) Cell cycle analysis with BrdU incorporation (12-hour pulse) and intracellular DAPI (icDAPI) staining for DNA content in E13.5 acd Lin-cKithi hematopoietic progenitors showing accumulation in G2/M phases of the cell cycle $(n=3$ mice per group from three independent experiments; mean $\pm \mathrm{SD}$ ). Representative flow cytometry plots are shown. Numbers indicate the percentage of cells in each gate. ${ }^{*} P<0.05 ;{ }^{* *} P<0.01 ;{ }^{* *} P<0.001$.

Recently, mutations affecting the shelterin gene TRF2 have been associated with aplastic anemia, and TINF2 mutations were identified in patients with a particularly aggressive form of DKC (24-29). As HSC loss leading to bone marrow failure is the most frequent cause of lethality in DKC, understanding the importance of telomerase and shelterin genes in hematopoiesis is relevant to human disease. Mice deficient for Acd due to homozygosity for a spontaneously arising hypomorphic acd allele demonstrate a pleiotrophic phenotype that includes adrenocortical dysplasia, caudal truncation, genitourinary abnormalities, skin hyperpigmentation, and substantial strain-dependent embryonic or perinatal lethality (30). These data indicate the tissue-specific functions of TPP1. Furthermore, the skin hyperpigmentation phenotype observed in acd/acd mice bears a striking similarity to that observed in DKC patients and in the POT1B-deficient mouse model. Subse- quent studies using complete loss of Acd in a skin-specific knockout model indicated that the hyperpigmentation phenotype resulted from functional defects in epidermal stem cells, suggesting a role for shelterin components in stem cell maintenance in this compartment (31). In both the complete knockout and hypomorphic Acd models, defects were linked to telomere dysfunction, triggering p53-mediated apoptosis and proliferative arrest (31-33).

To gain detailed insight into functions of the shelterin complex in hematopoiesis, we studied the impact of Acd deficiency on hematopoietic stem cells (HSCs). By targeting Acd, we focused on a central component of the shelterin complex that is essential for POT1A/B function and plays a role in telomerase recruitment. Our approach was designed to capture both the acute and long-lasting effects of Acd deficiency on HSCs and progenitor cells. We found that stem cell function was profoundly dependent on TPP1 in 
A BMT strategy

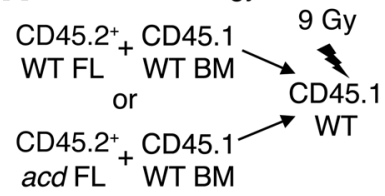

Experimental timeline

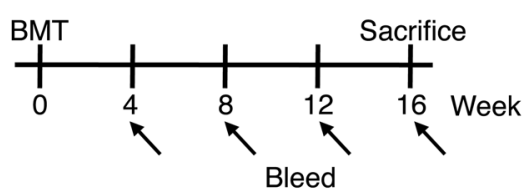

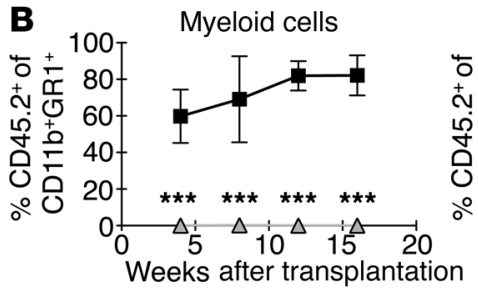
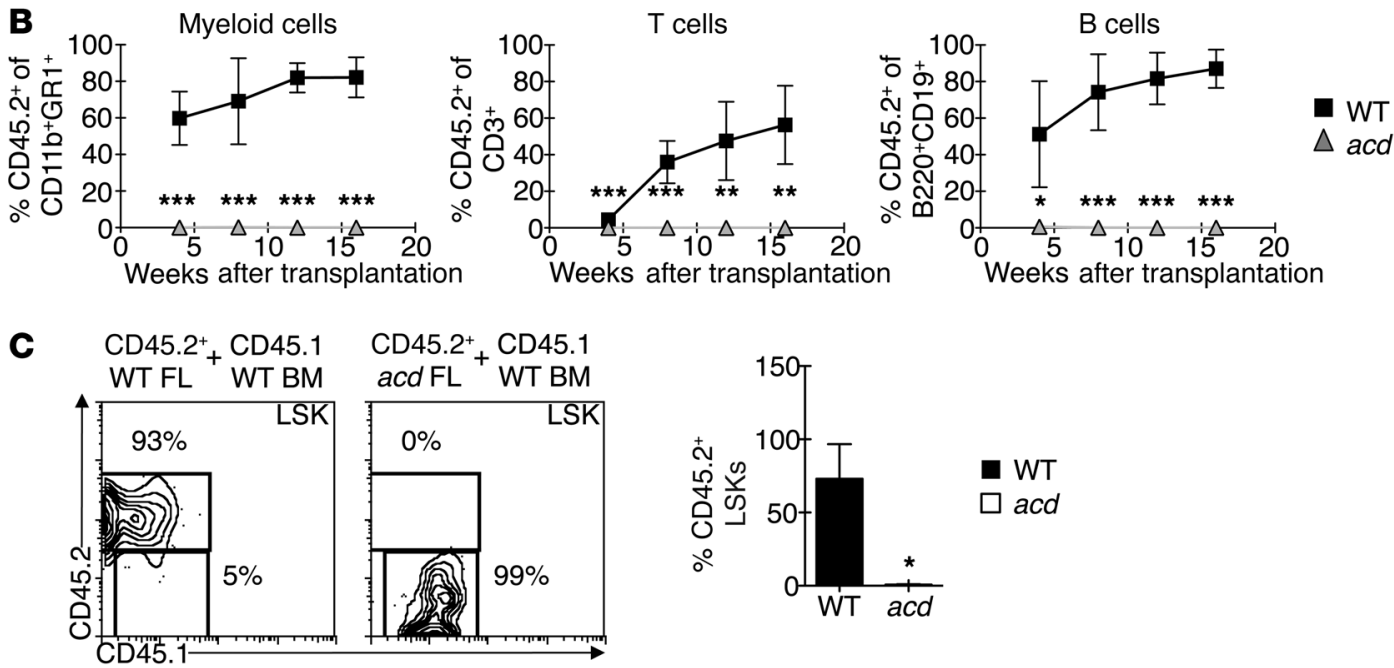

Figure 2

acd hematopoietic stem cells do not support long-term hematopoietic reconstitution. (A) Experimental design: lethally irradiated B6-CD45.1 mice were transplanted with 1:1 mixtures of E13.5 WT fetal liver (FL) (CD45.2+) or E13.5 acd hypomorphic FL (CD45.2+) and B6-CD45.1 competitor bone marrow $(\mathrm{BM})$ cells $\left(5 \times 10^{5}\right.$ each). (B) Flow cytometric analysis of peripheral blood 4-16 weeks after transplantation showing robust contribution of the WT but not the acd CD45.2+ FL graft to myeloid $(\mathrm{CD} 11 \mathrm{~b}+\mathrm{GR} 1+), \mathrm{T}$ cell $\left(\mathrm{CD} 3^{+}\right)$, and B cell compartments $\left(\mathrm{CD} 19^{+} \mathrm{B} 220^{+}\right)(n=3-5$ per group; mean \pm SD). (C) CD45.1/CD45.2 chimerism in Lin-Sca-1 ${ }^{\text {hicKithi }(L S K) ~ B M ~ p r o g e n i t o r s ~} 16$ weeks after transplantation. No residual CD45.2+ acd progenitors could be detected. Representative flow cytometry plots are shown. Numbers indicate the percentage of cells in each gate. Graphs show the mean \pm SEM. ${ }^{\star} P<0.05 ;{ }^{\star \star} P<0.01 ;{ }^{\star \star \star} P<0.001$. BMT, bone marrow transplantation.

both fetal and adult hematopoiesis. In mice homozygous for the hypomorphic acd allele, hematopoietic stem and progenitor cells were generated and maintained during fetal life, but they acquired phenotypic abnormalities, evidence of G2/M arrest, and a complete inability to reconstitute irradiated recipients after transplantation, indicating defective function. In the complete absence of $A c d$, HSCs were rapidly depleted, and animals progressed to frank hematopoietic failure. This phenotype was cell autonomous and surprisingly acute, as a complete depletion of the hematopoietic stem and progenitor compartment was observed within 5 days after Acd inactivation. TPP1 loss led to a rapid induction of p53 target gene expression. However, $p 53$ inactivation failed to rescue HSC depletion and function. These findings differed markedly from past observations of hematopoiesis in POT1B-deficient mice as well as of organ development in Acd-mutant mice and skin stem cell function in the absence of $A c d$, since all these phenotypes were largely rescued by $\mathrm{p} 53$ deficiency (31-34). Thus, our data identify an essential acute requirement for $A c d$ in hematopoietic stem and progenitor cells that differs from its effects in other tissues and stem cell compartments.

\section{Results}

Acd deficiency results in cell cycle arrest and impaired function of fetal liver HSCs. To assess the role of Acd in hematopoiesis, we first used mice that were homozygous for the spontaneously occurring hypomorphic acd allele (30). This allele was originally described to cause adrenocortical dysplasia and was thus named acd (30). It is characterized by a $G$ to $A$ transition within the third intron (Figure $1 \mathrm{~A}$ ), resulting in aberrant splicing and either a 7-bp insertion after exon 3 or inclusion of the third intron in the mRNA $(30,35)$. Both outcomes cause premature termination of translation and a truncated protein lacking all functional domains. Homozygosity for acd decreases the expression of WT transcripts to approximately $2 \%$ of normal levels, consistent with a profoundly hypomorphic phenotype (35). Analysis of the fetal livers from E13.5 acd-mutant mice revealed a mild decrease in fetal liver cellularity compared with that in the control littermates (Figure 1B). Of note, acd fetuses also had a reduction in body weight (not shown). When cell numbers were normalized in colony-forming assays measuring hematopoietic progenitor activity, no defect in the ability of acd fetal liver cells to form myeloid colonies was observed (Figure 1C). Flow cytometric analysis revealed that acd fetuses had a normal frequency of lineage- fetal liver cells and an increased frequency of lineage-Sca- $1^{+} \mathrm{cKit}{ }^{\text {hi }}$ (LSK) cells containing hematopoietic progenitors (Figure 1D). Furthermore, acd hypomorphism did not cause a significant defect in the overall frequency of CD $150^{+} \mathrm{CD} 48^{-}$ LSK cells, the most rigorous phenotypic definition of long-term HSCs (refs. 36, 37, and Figure 1D). Thus, HSC frequencies and myeloid progenitor activity were not compromised in acd animals. However, close analysis of the LSK progenitor compartment revealed that acd LSKs were larger, more granular, and expressed higher levels of 

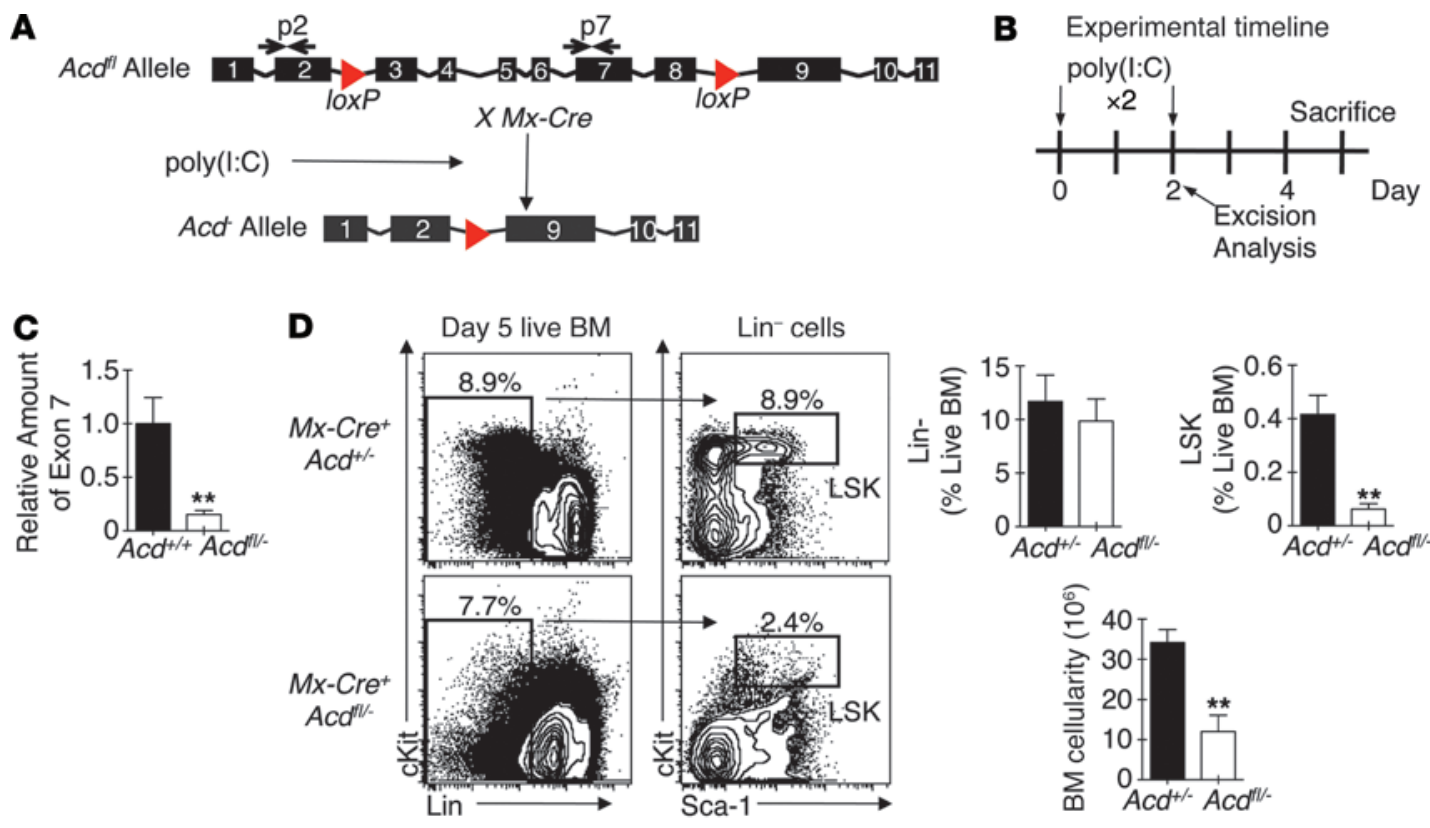

\section{Figure 3}

Acute depletion of adult hematopoietic progenitors after Acd inactivation. (A) Structure of the floxed Acd allele with loxP sites flanking exons 3-8 $\left(A c d^{f}\right) . M x$-Cre expression was achieved via poly $(\mathrm{l}: \mathrm{C})$ injections, leading to $A c d$ inactivation into an excised null allele $\left(\right.$ Acd $\left.^{-}\right)$. Arrows indicate $\mathrm{p} 2$ and p7 primer pairs used to quantify excision efficiency. (B) Experimental timeline to capture the acute effects of Acd inactivation. (C) qPCR for Acd exon 7 (p7) relative to exon 2 (p2) DNA in Lin-Sca- $1^{\text {hi }}$ cKithi (LSK) progenitors demonstrating approximately $80 \%$ excision of the floxed region after only a single poly $(\mathrm{I}: \mathrm{C})$ injection (qPCR; data are shown as the mean $\pm \mathrm{SEM}$ and are representative of three individual mice per group). (D) Flow cytometric analysis of hematopoietic progenitors on day 5 after poly $(\mathrm{I}: \mathrm{C})$ injection showing profound depletion of LSK progenitors and decreased BM cellularity $\left(\mathrm{Acd}^{+/-} n=5 ; A c d^{f / /-} n=4\right)$. Representative flow cytometry plots are shown. Numbers indicate the percentage of cells in each gate. ${ }^{* \star} P<0.01$.

Sca-1, suggesting an activated phenotype (Figure 1E). Cell cycle analysis demonstrated an accumulation of acd progenitors at the G2/M phases of the cell cycle (Figure 1F). This phenotype was reminiscent of the G2/M arrest reported in Acd-deficient embryonic fibroblasts and epidermal progenitor cells $(8,31)$.

Although colony formation assays measure progenitor function, they do not evaluate HSC self-renewal. To study the function of acd fetal HSCs, we performed competitive transplantation assays in lethally irradiated recipients and monitored long-term reconstitution (Figure $2 \mathrm{~A}$ ). We observed profound defects in trilineage reconstitution from acd fetal progenitors as early as 4 weeks after transplantation that persisted for the duration of the experiment (Figure 2B). When the LSK compartment was examined at the termination of the experiment, no contribution of acd cells could be detected (Figure 2C). These findings demonstrate that, despite preserved hematopoietic progenitor frequencies and myeloid progenitor activity, acd HSCs had severe functional impairment already fully apparent after primary transplantation.

Acd inactivation leads to acute depletion of adult hematopoietic stem and progenitor cells. Analysis of the acd hypomorphic phenotype revealed profound HSC functional defects, but nonhematopoietic developmental abnormalities in acd fetuses could have contributed to these defects through non-cell-autonomous mechanisms. Furthermore, analysis of a hypomorphic phenotype may underestimate the full impact of Acd deficiency, given the presence of residual WT transcripts at low levels. To bypass these limitations, we studied complete Acd inactivation in adult hematopoietic tissues using a conditional $A c d^{l}$ allele that we previously described to induce complete loss of function, including removal of POT1A/B from telomeres (ref. 8 and Figure 3A). This strategy facilitated robust, temporally controlled Acd excision and thus allowed us to study the acute effects of TPP1 loss in hematopoietic tissues. We induced $A c d^{f}$ inactivation using poly(I:C) injections to activate the IFN-responsive $M x$-Cre transgene (ref. 38 and Figure 3, A and B). Within 48 hours of a single injection, we achieved at least $80 \%$ excision of the floxed Acd allele (Figure 3C). Within 5 days, the hematopoietic progenitor compartment in $A c d l /$ - animals became severely depleted, and we could already detect a reduction in total bone marrow cellularity (Figure 3D). These data indicate that hematopoietic tissues are acutely sensitive to Acd inactivation and that this sensitivity can be traced back to a rapidly failing hematopoietic progenitor compartment.

The type I IFN response resulting from poly(I:C) administration was shown to drive quiescent HSCs into the cell cycle, which could enhance the impact of TPP1 loss in hematopoietic progenitors (39). To avoid this problem, we bred CreERT2 $\times A c d^{f l-}$ mice and inactivated $A c d$ with tamoxifen (ref. 40 and Supplemental Figure 1; supplemental material available online with this article; doi:10.1172/ JCI67871DS1). We observed decreased c-Kit expression on day 3 and the near-disappearance of LSK progenitors on day 5 after starting tamoxifen in the CreERT2 $\times A c d^{l /-}$ mice. These findings indicate that primitive hematopoietic progenitors are acutely sensitive to TPP1 loss, even in the absence of an IFN response. 

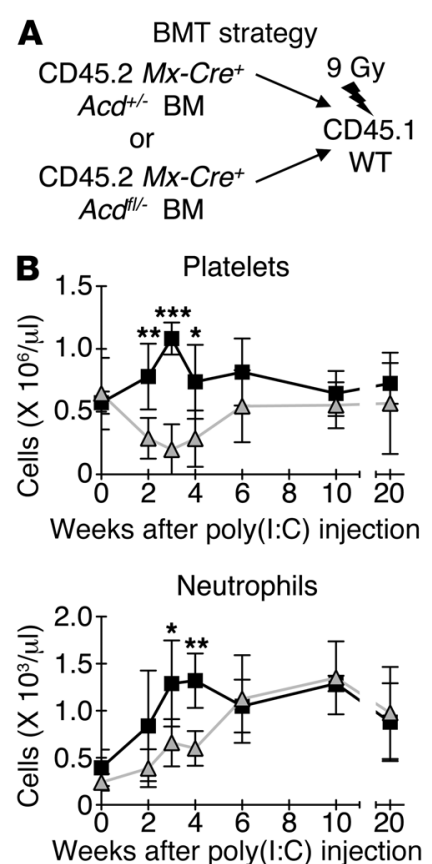
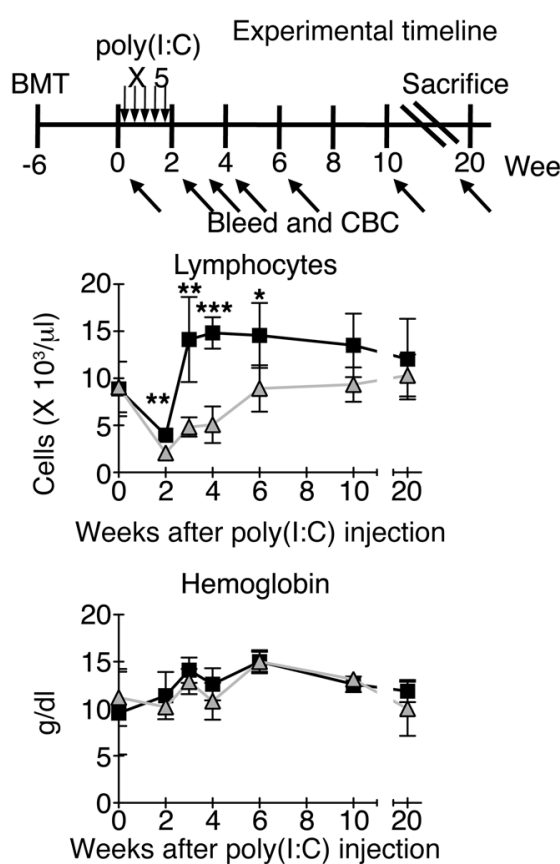
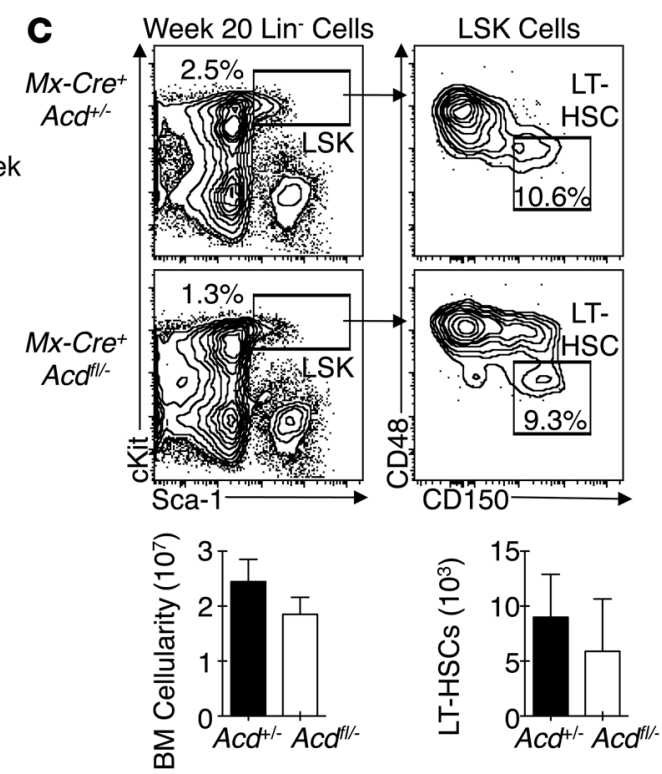

D

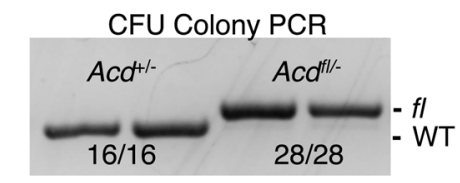

Colonies with indicated genotype per colonies screened

\section{Figure 4}

Hematopoietic inactivation of $A c d$ in a WT environment results in pancytopenia followed by strong selection for reconstitution by unexcised progenitors. (A) Experimental design: lethally irradiated B6-CD45.1 mice were transplanted with $\mathrm{Mx}-\mathrm{Cre}^{+} \mathrm{Acd} \mathrm{d}^{+-}$or $\mathrm{Mx}-\mathrm{Cre} \mathrm{Acd}^{+} \mathrm{Acl-} \mathrm{BM}$ cells $\left(5 \times 10^{5}\right)$. Six weeks later, poly(l:C) was administered to induce Acd inactivation only in donor-derived hematopoietic cells. (B) Complete blood counts (CBC) at baseline and 1-20 weeks after poly $(\mathrm{I}: \mathrm{C})$ injection showing a transient reduction in platelet, lymphocyte, and neutrophil counts (mean \pm SD). (C) Flow cytometric analysis of Lin-Sca-1 ${ }^{\text {hicKithi }}$ (LSK) progenitors and LT-HSCs 20 weeks after poly(l:C) injection, revealing no difference in HSC frequency or BM cellularity of $M x-C r e^{+} A c d^{+/-}$and $M x-C r e^{+} A c d^{f l l-}$ recipients $\left(A c d^{+/-} n=5 ; A c d^{f l /} n=3\right.$; mean $\left.\pm \mathrm{SEM}\right)$. Representative flow cytometry plots are shown. Numbers indicate the percentage of cells in each gate. (D) Clonal analysis of hematopoietic progenitors by GM-CFU and single-colony PCR at week 20. In $M x-C r e^{+} A c d^{f l /}$ recipients, all colonies analyzed (28 of 28) retained the $A c d^{f l}$ allele and had thus escaped $M x-C r e-m e d i a t e d ~ A c d$ inactivation. Representative PCR results are shown. ${ }^{\star} P<0.05$; ${ }^{\star \star} P<0.01 ;{ }^{* \star} P<0.001$.

Cell-autonomous mechanisms underlie the requirement for Acd in hematopoiesis. Although $M x$-Cre is primarily expressed in the bone marrow, activity can also be detected in other organs such as liver, kidney, and skin. Thus, non-cell-autonomous mechanisms could still contribute to hematopoietic defects in $\mathrm{Mx}-\mathrm{Cre}^{+} \times \mathrm{Acd}^{\mathrm{ll} /-}$ mice. To exclude this possibility, we generated bone marrow chimeras and studied Acd-deficient hematopoiesis in a WT environment. Lethally irradiated B6-CD45.1 mice were transplanted with $\mathrm{Mx}-\mathrm{Cr}^{+} \times \mathrm{Acd} \mathrm{d}^{\mathrm{ll} /-}$ or control $\mathrm{Mx}$ - $\mathrm{Cr} \mathrm{C}^{+} \times \mathrm{Acd}^{+/-}$bone marrow, allowing 6 weeks for full hematopoietic reconstitution before poly(I:C) injection (Figure 4A). To examine the effects of Acd inactivation on overall hematopoiesis, we monitored peripheral blood counts of transplant recipients for 20 weeks following poly(I:C) injection (Figure 4B). We found that TPP1 loss led to significant reductions in peripheral blood platelets, lymphocytes, and neutrophils between weeks 1 and 4 after poly(I:C) injection. Our subsequent analysis showed a recovery of peripheral blood counts by approximately 6 weeks. By week 20, bone marrow cellularity and LT-HSC numbers in $\mathrm{Mx}-\mathrm{Cre}^{+} \times \mathrm{Acd} / \mathrm{l} /$ - recipients had recovered to numbers similar to those in control bone marrow chimeras (Figure 4C). To identify the source of this reconstitution, we plated bone marrow in methylcellulose cultures, and individual colonies were harvested for clonal evaluation of Acd inactivation. All $\mathrm{Mx}$-Cre $\mathrm{C}^{+} \times \mathrm{Acd} f \mathrm{fl}$ - colonies analyzed (28 of 28) retained the floxed allele, demonstrating that reconstitution had occurred exclusively from cells escaping Cre-mediated gene deletion (Figure 4D).

In view of the strong selection pressure favoring cells that preserved an undeleted Acd allele, we generated competitive bone marrow chimeras in which CD45.2 ${ }^{+} \mathrm{Mx}-\mathrm{Cre}^{+} \times \mathrm{Acd}^{\mathrm{fl} /-}$ bone marrow could be studied in the presence of CD $45.1^{+}$WT competitors (Figure 5A). This strategy decreased the selection pressure favoring rare $\mathrm{Mx}$-Cre $\mathrm{C}^{+} \times \mathrm{Acd} \mathrm{fl}^{\mathrm{l}-}$ progenitors escaping Acd inactivation, as hematopoiesis was maintained by CD $45.1^{+}$competitors throughout the experiment. Bone marrow chimeras were given 6 weeks to facilitate the recovery of baseline hematopoiesis prior to Acd deletion. This strategy allowed us to study the effects of Acd deficiency on hematopoiesis after homing and engraftment had already occurred. Within 4 weeks of poly(I:C) administration, no contribution from the Acd-deficient CD $45.2^{+}$graft could 
A

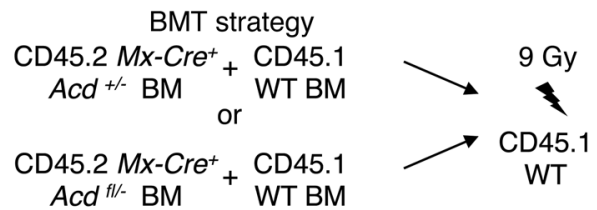

Experimental timeline

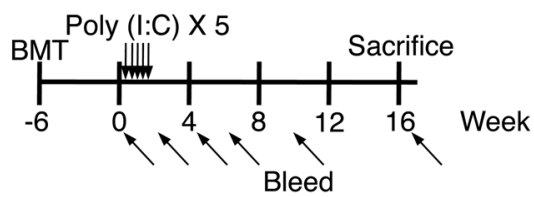

B

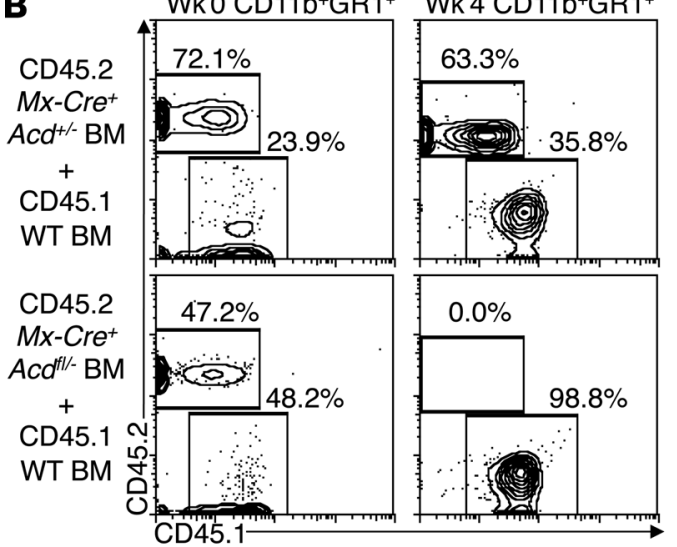

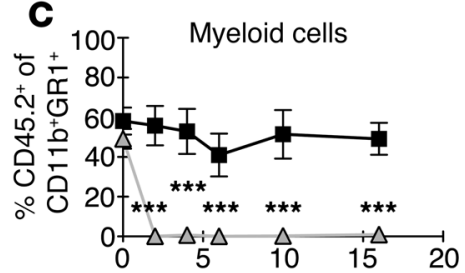

Weeks after poly $(\mathrm{I}: \mathrm{C})$ injection

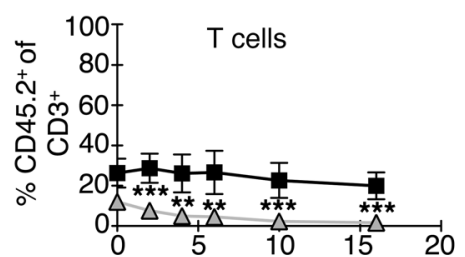

Weeks after Poly(I:C) injection

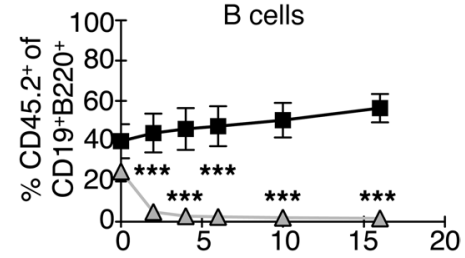

Weeks after poly $(\mathrm{l}: \mathrm{C})$ injection
- $\mathrm{Mx}-\mathrm{Cre}^{+} \mathrm{Acd}^{+-}$ $\triangle M x-\mathrm{Cre}^{+} \mathrm{Acd} \mathrm{d}^{\mathrm{t} / \mathrm{-}}$
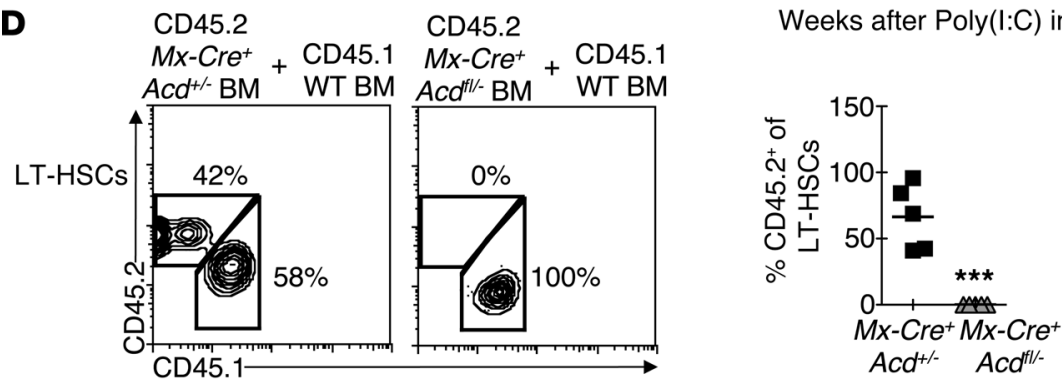

Figure 5

Acd-deficient HSCs do not support hematopoiesis after competitive BMT. (A) Experimental design: lethally irradiated B6-CD45.1 mice were transplanted with 1:1 mixtures of $M x-C r e+A c d^{+/-}$or $M x-C r e^{+} A c d^{f l l-}$ and competitor B6-CD45.1 BM cells $\left(5 \times 10^{5}\right.$ each). After 6 weeks to allow for hematopoietic reconstitution, baseline chimerism was assessed, followed by poly(l:C) administration. (B) Flow cytometric analysis of CD45.2/CD45.1 chimerism among CD11b+GR1+ blood myeloid cells at baseline (Wk 0) and 4 weeks after poly(I:C) (Wk 4) administration. Representative flow cytometry plots are shown ( $n=5$ mice per group from two independent experiments). (C) Flow cytometric analysis of peripheral blood 0-16 weeks after poly $(\mathrm{I}: \mathrm{C})$ injection showing a rapid drop in contribution of the CD45.2+ $\mathrm{Acd}^{\text {tll- }}$ graft to the myeloid $\left(\mathrm{CD} 11 \mathrm{~b}^{+} \mathrm{GR} 1^{+}\right), \mathrm{T} \mathrm{cell}\left(\mathrm{CD} 3^{+}\right)$, and $\mathrm{B}$ cell $\left(\mathrm{CD} 19+\mathrm{B} 220^{+}\right)$compartments (mean \pm SEM from two independent experiments). (D) CD45.2/CD45.1 chimerism in the CD150+CD48-Lin-Sca- $1^{\text {hi }}$ CKithi LT-HSC compartment 16 weeks after poly $(\mathrm{I}: \mathrm{C})$ administration revealing no residual CD45.2 ${ }^{+}$Acd ${ }^{f l-}$ LT-HSCs. Representative flow cytometry plots are shown ( $n=5$ mice per group). Numbers indicate the percentage of cells in each gate. ${ }^{\star \star} P<0.01 ;{ }^{* \star *} P<0.001$.

be detected among blood myeloid cells, a population with rapid turnover (Figure 5B). We found that peripheral blood $\mathrm{B}$ and $\mathrm{T}$ cells arising from the $A c d$-deficient graft also became progressively depleted (Figure 5C). This trilineage defect suggested the failure of multipotent stem and progenitor cells. Indeed, we were unable to detect any residual Acd-deficient HSCs at the termination of the experiment (Figure 5D). Acd ${ }^{+/-}$progenitors used in the control group competed well with an equivalent dose of B6-CD45.1 competitors, indicating the absence of a major hematopoietic defect after heterozygous Acd inactivation. Consistent with these findings, $A c d^{+/-}$mice had normal frequencies of LSK progenitors and phenotypically defined LT-HSCs (Supplemental Figure 2). Together, these data demonstrate that Acd is cell-autonomously required by HSCs, with strong selective pressure for rare progenitors that maintain a functional Acd gene to support hematopoietic recovery.
Acd inactivation in fetal liver hematopoietic stem and progenitor cells results in hematopoietic failure and death. Fetal and adult HSCs have numerous differences in gene expression programs, cytokine responsiveness, and cell cycle activity. Thus, it was possible that adult and fetal HSCs had different sensitivities to complete Acd inactivation. To address this question, we bred $A c d^{f / f l}$ mice with Vav-Cre transgenic mice (Figure 6A). Previous studies have demonstrated that Vav-Cre is expressed specifically in fetal hematopoietic cells starting at approximately E10.5, leading to near-complete excision of target loci by day E14.5 $(41,42)$. No $\mathrm{Vav}$-Cre $\mathrm{C}^{+} \times \mathrm{Acd} / \mathrm{flfl}$ mice were born, while other genotypes were present at expected Mendelian frequencies (Table 1). At E14.5, live $\mathrm{Vav}-\mathrm{Cr}^{+} \times A c d^{f / f l}$ fetuses were present, but these mice had pale fetal livers and vasculature, suggesting defective hematopoiesis (Table 2 and Figure 6B). Flow cytometric analysis revealed that fetuses with hematopoietic-specific Acd inactivation had few, if any, residual hematopoietic LSK progenitors in the liver 
A

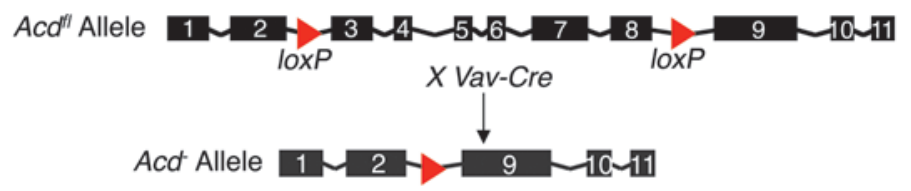

Live births

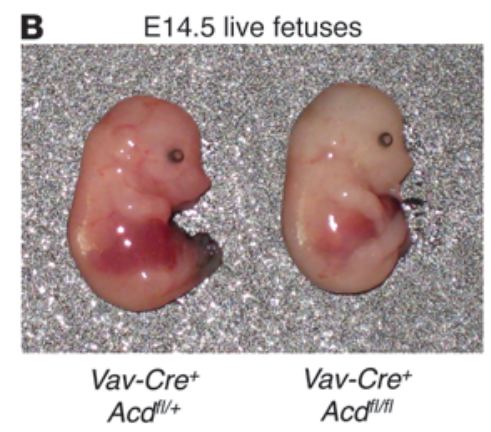

\section{Figure 6}

Complete Acd inactivation in fetal hematopoietic cells is incompatible with survival. (A) Experimental design: Acd inactivation restricted to fetal hematopoiesis was achieved using a Vav-Cre ${ }^{+}$transgene. Breedings were established using Vav-Cre ${ }^{+} \mathrm{Acd} \mathrm{CH}^{\mathrm{fl}+}$ parents. (B) At E14.5, live Vav-Cre+Acd ${ }^{f / f l}$ fetuses could be identified, however, these mice were pale compared with those of all other genotypes. (C) Flow cytometric analysis of E14.5 fetal livers demonstrating that $\mathrm{Vav}-\mathrm{Cre}^{+} \mathrm{Acd} \mathrm{d}^{\mathrm{flfl} l}$ fetuses had profoundly depleted $\mathrm{Lin}-\mathrm{Sca}-{ }^{+}{ }^{+} \mathrm{CKit}{ }^{\text {thi }}$ (LSK) hematopoietic progenitors. Representative flow cytometry plots are shown. Numbers indicate the percentage of cells in each gate. at E14.5 (Figure 6C). These data demonstrate that maintenance of both fetal and adult HSCs is acutely dependent on Acd.

Acd deficiency results in acute $p 53$ activation, but $p 53$-independent cell cycle arrest, in hematopoietic progenitors. To determine whether p53 activation occurs in hematopoietic progenitors following Acd inactivation, we assessed the expression of the p53 target genes Puma, Noxa, and $p 21$ within days after the induction of Cre recombinase, but before hematopoietic progenitors were lost (Figure 7A). Acd deletion resulted in the upregulation of Noxa and p21 (Figure 7B), but not Puma (not shown), within 48 hours of poly(I:C) treatment. This effect was p53 dependent, as LSK progenitors from mice deficient for both Acd and $p 53$ did not demonstrate upregulated expression of $p 21$ or Noxa. Consistent with these observations, we detected the accumulation of p53 protein in cultured CreERT2 $\times A c d^{f l /-}$ bone marrow progenitors exposed to 4-hydroxy-tamoxifen (not shown). Next, we assessed the cell cycle status of LSK progenitors shortly after Acd inactivation in the presence or absence of p53 (Figure 7, C and D). Upon TPP1 loss, we observed an accumulation of hematopoietic progenitors in the G2/M phases of the cell cycle, consistent with the presence of G2/M arrest. Interestingly, we observed this cell cycle arrest even in the absence of $\mathrm{p} 53$. These data indicate that while p53 activation occurs acutely following Acd deletion, it is not required for G2/M arrest.
Acd deficiency results in chromosomal fusions at telomeres in the absence of detectable telomere shortening. TPP1-deficient embryonic fibroblasts display an increased rate of rare chromosomal fusion events involving telomeres. To evaluate whether this occurred in hematopoietic tissues, we prepared metaphase spreads from control or $\mathrm{Mx}$-Cre $\mathrm{C}^{+} \times \mathrm{Acd} / \mathrm{l} / \mathrm{-}$ bone marrow cells within 48 hours after poly(I:C) treatment (Figure 8A). This strategy ensured that dividing hematopoietic progenitors could be examined acutely after Acd inactivation, while their numbers were still preserved. Efficient Cre-mediated excision was detected (Figure 8B). Quantitative PCR (qPCR) showed that the amount of telomere signal was largely preserved at this stage in $\mathrm{Mx}-\mathrm{Cr} e^{+} \times \mathrm{Acd} \mathrm{d}^{\mathrm{l} / \mathrm{-}}$ bone marrow, consistent with the absence of major telomere shortening within days of TPP1 loss (Figure 8C). In contrast, FISH analysis revealed an increased frequency of metaphases in which we detected chromosomal fusions with a shared telomeric signal (Figure 8D and Table 3 ). These findings are consistent with TPP1 loss driving the fusion of deprotected telomeric ends in dividing hematopoietic progenitors.

p53 inactivation does not rescue the function and maintenance of Acd-deficient HSCs. Previous studies demonstrated that $p 53$ inactivation rescued many features of abnormal development in hypomorphic acd mice $(32,33)$. Furthermore, p53 deficiency restored epidermal stem cell function following Acd inactivation (31). To investigate whether p53 inactivation could rescue HSC func-

Table 1

Acd deletion in utero is incompatible with live birth

\begin{tabular}{|c|c|c|c|c|c|c|c|}
\hline acd & $+/+$ & $f l /+$ & $f l / f l$ & $+/+$ & $f l /+$ & $f l / f l$ & Total \\
\hline Vav-Cre & - & - & - & + & + & + & \\
\hline Total & 9 & 14 & 4 & 18 & 16 & 0 & 61 \\
\hline Predicted & 3.8125 & 7.625 & 3.8125 & 11.4375 & 22.875 & 11.4375 & 61 \\
\hline
\end{tabular}

Genotyping results demonstrating that no Vav-Cre+Acd flffl mice were born, while all other genotypes were represented. Genotyping results for this group varied significantly from Mendelian predictions by $\chi^{2}$ analysis $\left(P=1.60 \times 10^{-5}\right)$. 
A

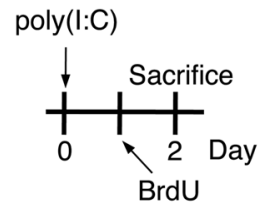

B

p21

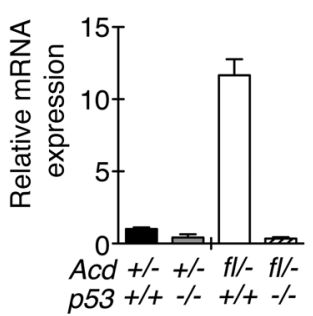

Noxa

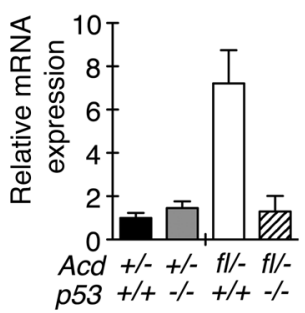

C
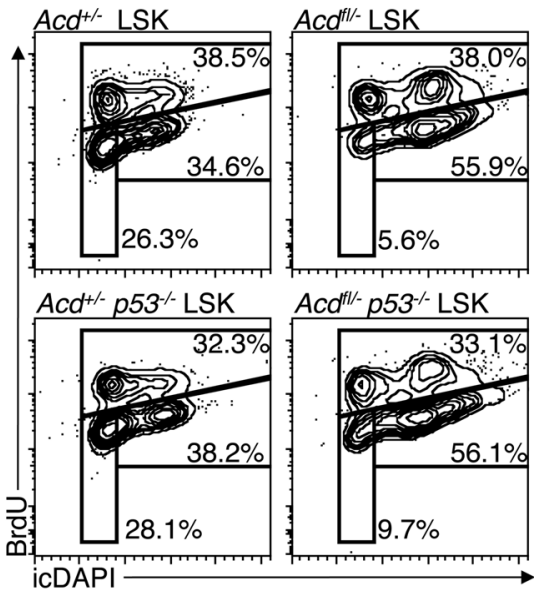

D

LSK cell cycle

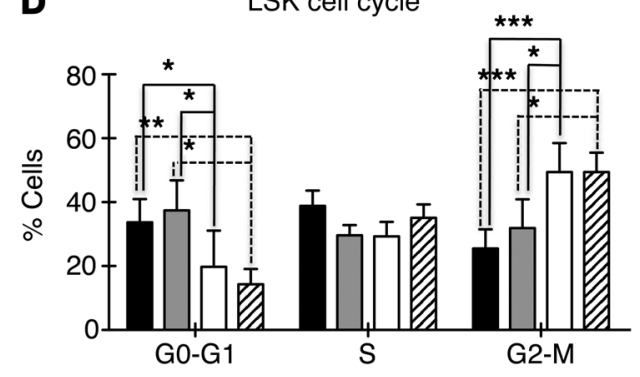

Figure 7

Acd inactivation results in acute cell cycle arrest and induction of p53 target genes. (A) Experimental design: Mx-Cre Acd $^{+/-}$, Mx-Cre Acd $^{+/-} p 53^{-/-}$, $M x-C r e^{+} A c d^{f l-}$, and $M x-C r e^{+} A c d^{f l l}-p 53^{-1-}$ mice were injected with a single dose of poly(I:C) followed by BrdU and sacrificed as indicated. (B) Relative abundance of p21 and Noxa transcripts in purified Lin-Sca-1 ${ }^{\text {hicKithi }}$ (LSK) progenitors demonstrating that Acd deletion induced a p53-dependent increase in p21 and Noxa mRNA (qRT-PCR; data are shown as the mean \pm SEM and are representative of at least two independently sorted samples per group). (C) Flow cytometric analysis of progenitors for BrdU incorporation and icDAPI showing the accumulation of $\mathrm{Mx}-\mathrm{Cre}^{+} \mathrm{Acd}^{\mathrm{fll}-}$ and $\mathrm{Mx}-\mathrm{Cre}^{+} \mathrm{Acd}^{\mathrm{fll}-} \mathrm{p} 53^{-/-}$ cells in G2/M phases of the cell cycle. Representative flow cytometry plots are shown from four independent experiments. (D) Quantification of data are shown in $\mathbf{C}$ ( $n=3$ mice per group). ${ }^{*} P<0.05 ;{ }^{* *} P<0.01 ;{ }^{* *} P<0.001$. tion and maintenance following Acd deletion, we generated hematopoietic chimeras with WT CD $45.1^{+}$bone marrow and bone marrow from CD $45.2^{+} \mathrm{Mx}-\mathrm{Cre}^{+} \times \mathrm{Acd}^{+/-}$or $\mathrm{Mx}-\mathrm{Cr} \mathrm{e}^{+} \times \mathrm{Acd} \mathrm{d}^{\mathrm{ll} /-}$ mice, in the presence or absence of $p 53$ (Figure 9A). After hematopoietic reconstitution, poly(I:C) was administered to induce $M x$-Cre expression and Acd inactivation specifically in the cohort of CD $45.2^{+}$hematopoietic cells. Mice transplanted with either $\mathrm{Mx}-\mathrm{Cr} \mathrm{e}^{+} \mathrm{Ac} \mathrm{dfl}^{\mathrm{fl}-} \mathrm{p} 53^{+/+}$or $\mathrm{Mx}-\mathrm{Cr} \mathrm{e}^{+} \mathrm{Acd} \mathrm{d}^{\mathrm{fl} /-} \mathrm{p53^{-/- }}$ progenitors lost all myeloid cells originating from the CD45.2+ graft within 2 weeks of Acd inactivation and at all subsequent time points (Figure 9B). Additionally, CD 45.2 ${ }^{+}$Acd-deficient $\mathrm{T}$ and $\mathrm{B}$ cells were progressively lost, irrespective of $\mathrm{p} 53$. Sixteen weeks after poly(I:C), flow cytometric analysis of long-term HSCs demonstrated that Acd-deficient HSCs were completely depleted, even in the presence of $p 53$ inactivation (Figure 9C). In fact, our analysis of the bone marrow revealed that the numbers of Acd-deficient HSCs were already profoundly reduced within 1 week of Acd inactivation, regardless of $p 53$ status (Supplemental Figure 3). Altogether, p53 activation occurred acutely after loss of TPP1 in hematopoietic progenitors. However, unlike in epider-

\section{Table 2}

Acd-deficient embryos are viable at E14.5

$\begin{array}{lccccccc}\text { acd } & +/+ & f l /+ & f l / f l & +/+ & f l /+ & f l / f l & \text { Total } \\ \text { Vav-Cre } & - & - & - & + & + & + & \\ \text { Total } & 3 & 2 & 0 & 8 & 6 & 4 & 23 \\ \text { Predicted } & 1.4375 & 2.875 & 1.4375 & 4.3125 & 8.625 & 4.3125 & 23\end{array}$

No difference was detected between the observed genotypes and those predicted by Mendelian ratios $(P=0.19)$. mal stem cells and other cellular compartments, the maintenance and function of Acd-deficient HSCs could not be rescued by p53 inactivation.

Acd-deficient hematopoietic progenitors undergo p53-independent caspase- $3 / 7$ activation and show evidence of aneuploidy. Since Acd deficiency resulted in acute depletion of hematopoietic stem and progenitor cells, we explored cell death as a potential mechanism contributing to this phenotype. To examine apoptosis, we determined the abundance of activated caspases 3 and 7 that execute this form of cell death, a reliable assay to detect apoptosis in hematopoietic cells (43). We found significant caspase-3/7 activation in both $p 53^{+/+}$ and $p 53^{-/-}$bone marrow cells within 48 hours of Acd inactivation (Figure 10, A and B). To assess whether necrosis also contributed to acute depletion, we measured HMGB1 levels in the serum of the same animals used for the apoptosis assay. HMGB1 is a nuclear protein released by cells during necrosis but not apoptosis (44). ELISA analysis revealed no difference in HMGB1 levels between the sera of WT and Acd-deficient mice (Figure 10C). Together, these data suggest that hematopoietic cell depletion arose from apoptosis and not necrosis following acute Acd inactivation, but this occurred independently of p53 activation.

To further explore the mechanisms leading to caspase-3/7 activation, we assessed mitochondrial mass and potential in Acd-deficient progenitors. Previous reports have identified that shelterin dysfunction and telomere instability can induce mitochondrial compromise $(45,46)$. Flow cytometry showed a trend toward increased mitochondrial mass in Acd-deficient LSK progenitors (Figure 10D). This type of mitochondrial accumulation is consistent with cell cycle arrest, as described in Figure 7. We next examined mitochondrial membrane potential as a read-out for mitochondrial function, an analysis that revealed increased mitochondrial 
A

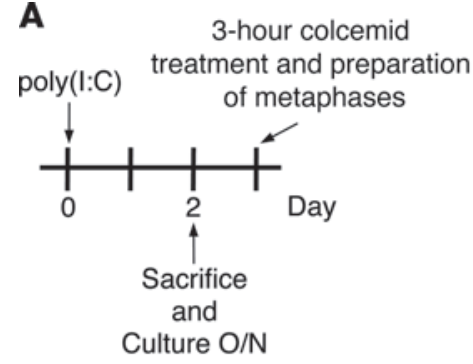

D

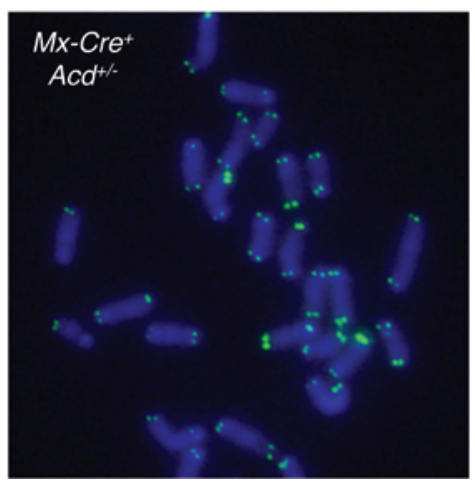

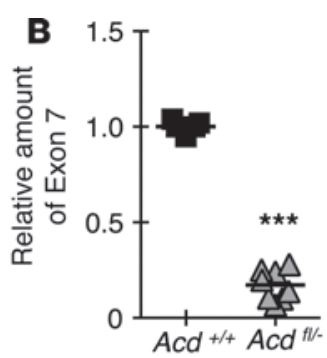
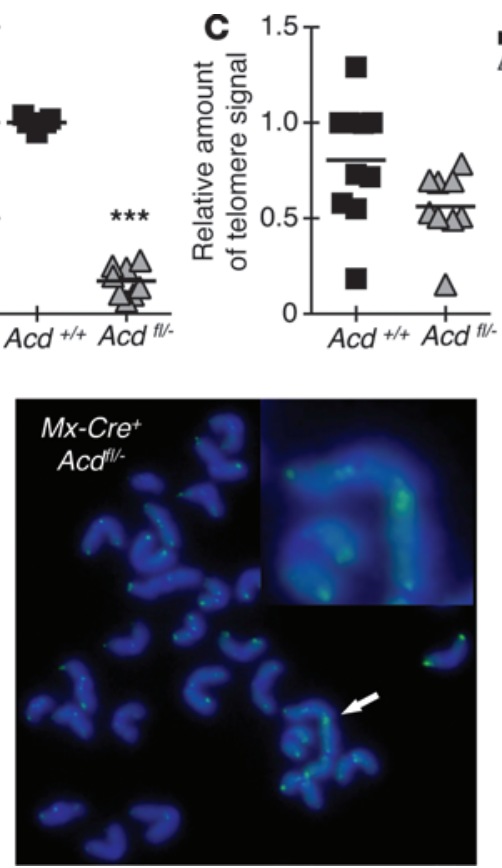

Mx-Cre+Acd ${ }^{* / *}$ $\triangle M x-C r e^{+} A c d^{m-1}$

Acd-deficient hematopoietic progenitors demonstrate acute chromosomal instability in the absence of telomere shortening. (A) Experimental design: BM was harvested from $\mathrm{Mx}-\mathrm{Cre}^{+} \mathrm{Acd}^{+/-}$or $\mathrm{Mx}-\mathrm{Cre}^{+} \mathrm{Acd}^{\mathrm{fl} / \mathrm{-}}$ mice 48 hours after a single dose of poly $(\mathrm{I}: \mathrm{C})$ and cultured overnight with IL-3/IL-6/SCF. Metaphases were prepared after a 3-hour colcemid treatment. (B) Relative abundance of exon 7 DNA signal normalized to exon 2 showing rapid Cre-mediated excision in total BM $\left(A c d^{+/+}: n=7 ; A c d^{f l /-}: n=8\right)$. ${ }^{* *} P<0.001$. (C) qPCR assessment of telomere length in BM cells acutely (48-60 hours) after Acd inactivation $\left(\mathrm{Acd}^{+/+}: n=10 ; A c d^{\mathrm{fl} / \mathrm{-}}\right.$ : $n=9$ ) (p.0613). (D) Representative images of metaphases stained with DAPI and an FITC-labeled telomeric probe. Insert shows a chromosomal fusion event centered on a telomeric signal. Original magnification, $\times 1,000(\times 4,300$, insert $)$. membrane potential following Acd inactivation (Figure 10E). These data are consistent with the accumulation of normally functioning mitochondria. Together, these findings suggest that Acd inactivation did not cause acute mitochondrial damage and that p53-independent caspase-3/7 activation did not occur via the intrinsic mitochondrial pathway of apoptosis.

Genomic instability can result in an abnormal progression through mitosis and the generation of aneuploid cells. Broad, sustained genomic instability can result in mitotic catastrophe through caspase activation and robust apoptosis via p53-dependent and p53-independent mechanisms $(47,48)$. We considered this possibility in $A c d$-deficient hematopoietic stem and progenitor cells, as previous reports in MEFs demonstrated that Acd inactivation resulted in the accumulation of aneuploid cells, a hallmark of abnormal mitosis (8). We examined DNA content in $p 53^{+/+}$and $p 53^{-/-}$ LSK progenitors within 48 hours of Acd inactivation and observed a significant increase in cells with greater than 4 N DNA content after acute Acd inactivation, regardless of p53 status (Figure 10F). This finding suggested that progression to mitotic catastrophe in Acd-deficient hematopoietic progenitor cells could contribute to HSC loss $(47,48)$. In addition, the extrinsic pathway could have contributed to caspase-3/7 activation independently of p53 and mitochondrial dysfunction (49-51). Altogether, Acd deletion was sufficient to trigger p53 activation, but p53 was not required to induce massive cell death and severe hematopoietic failure.

\section{Discussion}

Our findings demonstrate that HSCs are highly sensitive to shelterin deprotection initiated by Acd inactivation. Complete inactivation of the Acd gene induced a surprisingly acute depletion of the fetal or adult blood-forming stem cell compartments, leading to rapid hematopoietic failure before telomere shortening could be detected. Although TPP1 and the shelterin complex play dual functions in recruiting telomerase and shielding telo- meric ends from an uncontrolled DNA damage response, our observations indicate that the overall effects of shelterin dysfunction in hematopoietic progenitors are dominated by acute telomere deprotection. TPP1-deficient HSCs rapidly upregulated p53 target gene transcription and had evidence of cell cycle arrest and chromosomal instability. Surprisingly, however, the maintenance of TPP1-deficient stem cells was not rescued by $p 53$ inactivation, and these cells showed p53-independent activation of caspase-3/7. These findings contrast with the major effects of TPP1 loss on epidermal stem cell homeostasis and fetal skeletal development, which are rescued in the absence of p53 (31-33). Even without complete loss of TPP1, as modeled by homozygosity for the hypomorphic acd allele, HSC function was severely impaired. This demonstrates that blood-forming stem cells are very sensitive to defects in telomere protection as compared with other tissues.

Telomere deprotection has been shown to trigger a p53/p21-dependent cell cycle arrest (52). Similarly, p53 activation contributes to cellular senescence in response to critical telomere shortening (53-55). These findings, combined with data demonstrating that $p 53$ inactivation rescued epidermal stem cell defects owed to Acd inactivation, led us to investigate the role of $p 53$ in bone

\section{Table 3}

Acd-deficient BM cells have an increase in chromosomal fusions

\begin{tabular}{lcc} 
Genotype & $\begin{array}{c}\text { Metaphases } \\
\text { analyzed }\end{array}$ & $\begin{array}{c}\text { Chromosome fusions } \\
\text { plus telomere signal }\end{array}$ \\
$\mathrm{Mx}-\mathrm{Cre}^{+} \mathrm{Acd} d^{+/-}$ & 49 & $3(6.1 \%)$ \\
$\mathrm{Mx}-\mathrm{Cre}^{+} \mathrm{Acd^{f// } -}$ & 30 & $5(16.7 \%)$ \\
\hline
\end{tabular}

Blind scoring of metaphases demonstrated an increase in chromosome fusions containing a telomere signal among $\mathrm{Mx}-\mathrm{Cre}^{+} \mathrm{Acd} \mathrm{fll}^{\mathrm{fl}}$ metaphases. 
A

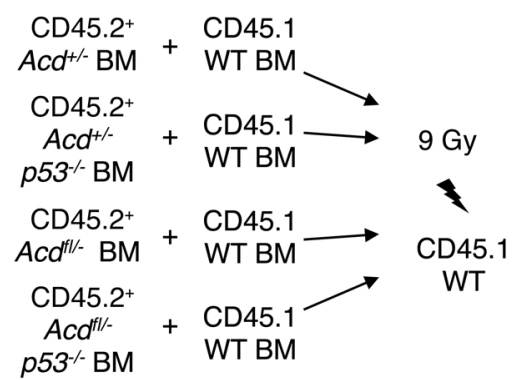

Experimental timeline

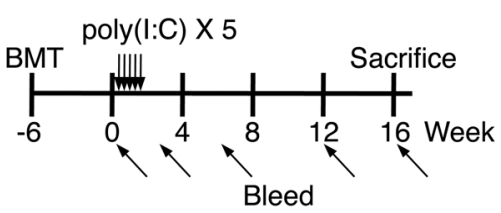

B

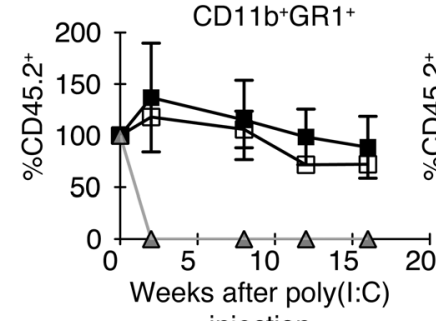

injection

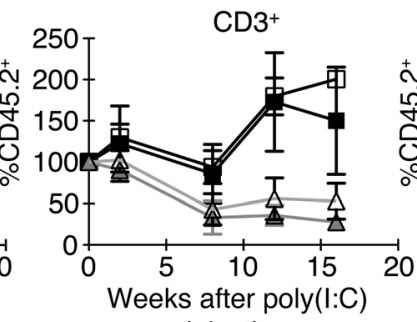

injection

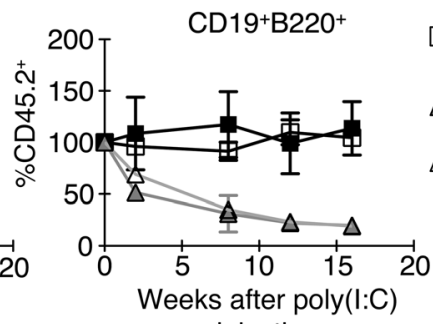

injection

C
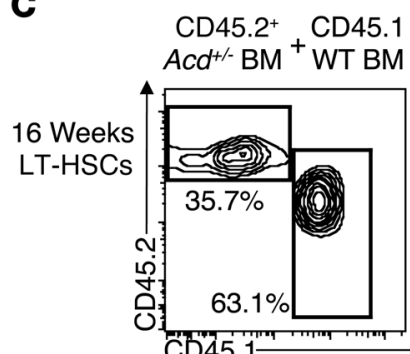

CD45.2 $2^{+}$

$\mathrm{Acd}^{+-}+\mathrm{CD} 45.1$ p53\% BM WT BM Acd $^{\text {th/ } / \text { BM }}{ }^{+}$WT BM

CD45.2 ${ }^{+}$

Acd fil- + CD45.1

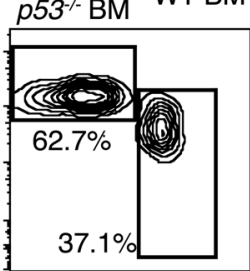

- $\mathrm{Mx}-\mathrm{Cre}^{+} \mathrm{Acd}^{+1-}$

$\square \mathrm{Mx}-\mathrm{Cre}^{+} \mathrm{Acd}^{+/-}$ p53-

$\triangle \mathrm{Mx}-\mathrm{Cre}^{+} \mathrm{Acd}^{\mathrm{fl} / \mathrm{-}}$

$\triangle M x-C r e^{+} A c d^{f i l-}$ p53\%

\section{Figure 9}

p53 inactivation does not rescue the survival and function of $A c d$-deficient HSCs. (A) Experimental design: lethally irradiated B6-CD45.1 mice were

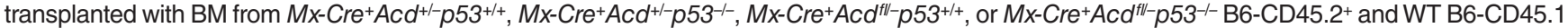
competitor mice $\left(5 \times 10^{5}\right.$ cells each, $1: 1$ ratio). After 6 weeks to allow for hematopoietic reconstitution, poly(I:C) was administered. (B) Flow cytometric analysis of peripheral blood 2-16 weeks after poly(I:C), administration showing a rapid drop in contribution of the $\mathrm{CD}^{2} 5.2^{+} \mathrm{Mx}^{-C r e} \mathrm{C}^{+} \mathrm{Acd^{fl } -}-\mathrm{p} 53^{+/+}$and

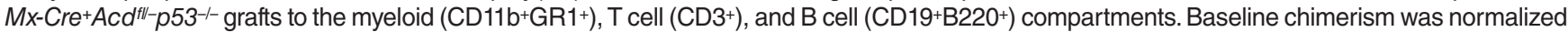
to $100 \%$ in each mouse. The percentage of $\mathrm{Mx}-\mathrm{Cre}^{+} \mathrm{Acd} \mathrm{fll}^{\mathrm{fl}} \mathrm{p5} 3^{+/+}$and $\mathrm{Mx}-\mathrm{Cre}^{+} \mathrm{Acd^{fl- }} p 53^{-/-}$graft output differed significantly from $\mathrm{Mx}-\mathrm{Cre}{ }^{+} \mathrm{Acd} \mathrm{C}^{+/-} p 53^{+/+}$ and $\mathrm{Mx}-\mathrm{Cre}{ }^{+} \mathrm{Acd}^{+/-} \mathrm{p53^{-/- }}$ output in the myeloid compartment by week $2(P<0.001)$, in the T cell compartment by week $12(P<0.01)$, and in the B cell compartment by week $8(P<0.01)$. Data represent the mean \pm SD $(n \geq 4$ per group). (C) CD45.2/CD45.1 chimerism in CD150+CD48-Lin-Sca-1 hicKithi LT-HSCs 16 weeks after poly(I:C) administration, showing no residual CD45.2+ $M x-C r e^{+} A c d^{f l l-} p 53^{+/+}$or $M x-C r e^{+} A c d^{f l l-} p 53^{-/-} H S C s$. Representative flow cytometry plots are shown. Numbers indicate the percentage of cells in each gate.

marrow failure in the setting of Acd deficiency (31). Surprisingly, we found that while Acd inactivation resulted in increased transcription of p53 targets, indicating p53 activation, p53 deficiency did not rescue any component of the hematopoietic stem and progenitor cell defects that we tested. Instead, we observed p53-independent activation of the executioner caspases 3 and 7, with no evidence of necrotic cell death. Because caspase activation occurred without mitochondrial depolarization, our findings suggest the activation of the extrinsic pathway of apoptosis or an alternative pathway related to defective mitosis and aneuploidy, both of which can be p53 independent (47-51). Altogether, these data indicate that p53 activation is not the predominant underlying cause of HSC functional defects or bone marrow failure following Acd deletion and suggest that different stem cell compartments such as HSCs and skin stem cells may respond very differently to shelterin dysfunction. HSCs, but not epidermal progenitors, were sensitive to a $\mathrm{p} 53$-independent form of apoptosis after Acd inactivation, which could indicate either differential induction of or differential sensitivity to this p53-independent pathway. Altogether, our observations highlight the fact that model systems (e.g., fibroblast cultures) cannot fully predict the biological roles of individual shelterin components in vivo and that direct comparisons of different tissues are essential.

Telomere dysfunction has been hypothesized to play a role in multiple contexts including organ failure, aging, and cancer. $\mathrm{DKC}$ is the prototypical human disorder most directly associated with defects in telomere homeostasis and is a hereditary syndrome characterized by progressive telomere shortening. Although bone marrow failure is the most significant organ dysfunction and the leading cause of death in DKC, affected patients can also develop abnormalities in the skin, mucosal tissues, biliary tract, and lungs, suggesting that multiple somatic stem cell compartments are affected. In mice with reduced expression of 
A

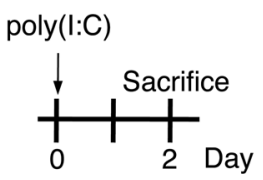

D

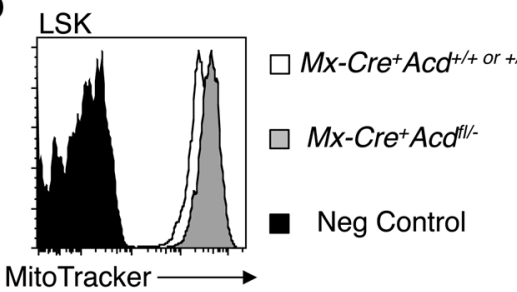

E LSK

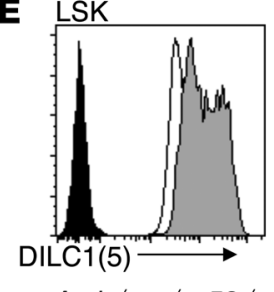

F $\mathrm{Acd}+1+$ or $+/ p 53^{+/+}$

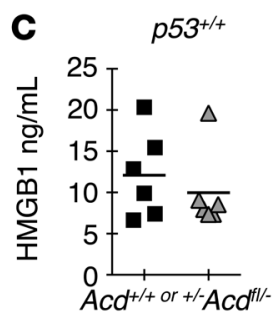

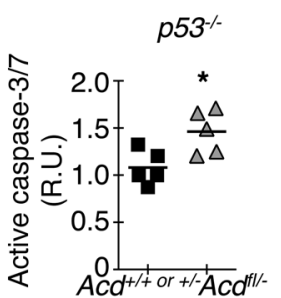

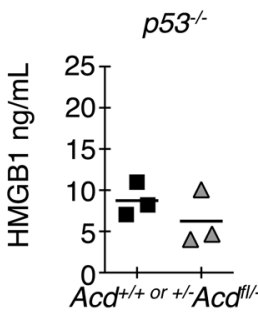

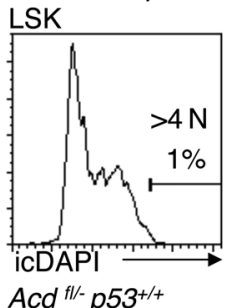

LSK

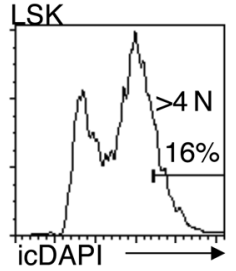

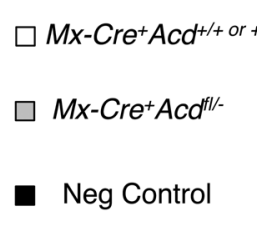

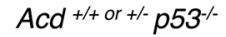
LSK

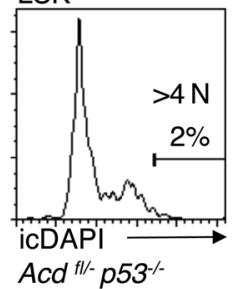

LSK

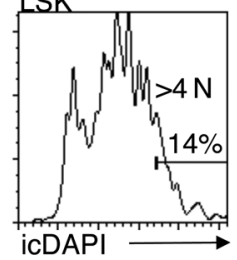

Mitochondrial mass

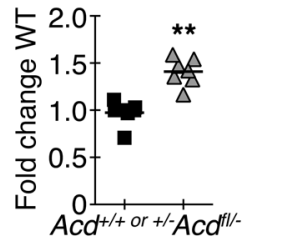

Membrane potential

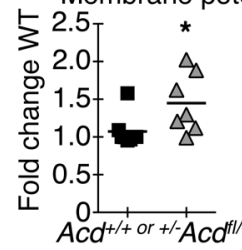

$A c d^{+1++o r+1-A c d^{(t / 1 /-}}$

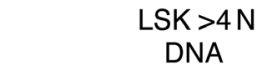

\section{Figure 10}

Acd inactivation results in the induction of $p 53$-independent caspase-3/7 activation and aneuploidy. (A) Experimental design: BM was harvested from $\mathrm{Mx}-\mathrm{Cre}^{+} \mathrm{Acd}^{+/+}$or ${ }^{+/-}$or $\mathrm{Mx}$-Cre+ $\mathrm{Acd}^{\mathrm{fl}-}$ mice 48 hours after a single dose of poly(I:C). (B) Luminescence-based analysis of caspase-3/7 activation in the BM demonstrating a significant increase in activation in $M x-C r e^{+} A c d^{f l l}-$ mice, regardless of $p 53$ status, compared with the indicated controls. Data points represent the mean of technical triplicates from individual mice ( $n \geq 5$ per group). (C) ELISA analysis demonstrating no difference in HMGB1 serum levels between $\mathrm{Mx}-\mathrm{Cre}^{+} \mathrm{Acd^{fl/ } -}$ mice and the indicated controls, regardless of $p 53$ status. Data points represent the mean of technical triplicates from individual mice ( $n \geq 3$ per group). (D) Flow cytometric analysis of mitochondrial mass using MitoTracker in phenotypic LSK progenitors showing a significant increase in mass in $\mathrm{Mx}-\mathrm{Cre}^{+} \mathrm{Acd} \mathrm{d}^{\mathrm{fl}-}$ mice compared with controls. (E) Flow cytometric analysis of mitochondrial membrane potential using DilC1(5) in phenotypic LSK progenitors showing a significant increase in membrane potential in $M x$-Cre+Acd ${ }^{f l l-}$ mice compared with controls. (F) Flow cytometric analysis of DNA content showing an accumulation of LSK progenitor cells with greater than $4 \mathrm{~N}$ DNA

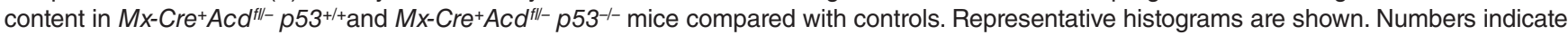
the percentage of cells in each gate. ${ }^{*} P<0.05$; ${ }^{* \star} P<0.01$; ${ }^{\star \star} P<0.001$ compared with control, matched for $p 53$ status where applicable.

Terc or Tert genes, progression to stem cell loss typically occurs only after telomere attrition accumulates through several successive mouse generations due to the fact that the mice have substantially longer telomeres than humans (3). With respect to shelterin components, a mouse syndrome reminiscent of DKC was first reported using combined Pot $1 b$ deficiency and Terc haploinsufficiency $(22,34,56)$. In this model, a relatively mild DNA damage response and gradual telomere shortening were identified as drivers of hematopoietic failure as well as of gradual homeostatic dysfunction in rapidly proliferating tissues. However, the significance of this model for human disease is limited because POT1b is a mouse-specific shelterin component $(20,21)$. Interestingly, shelterin dysfunction has recently been linked to human bone marrow failure, with the identification of TRF2 mutations in aplastic anemia and TINF2 mutations in a severe form of DKC that exhibited exceptionally short telomeres and early onset (24-29). The acute nature of this form of DKC might be due to the fact that TINF2 mutations, like our Acd inactivation model, result in rapid telomere deprotection and destabilization, rather than the erosion observed in the Pot $1 b$ mouse model and in DKC patients with TERC or TERT mutations. In fact, data from MEFs indicate that the critical function of TIN2 is to stabilize the TPP1-POT1A/B complex at telomeres (16). The progression toward stem cell loss may thus be markedly accelerated in the presence of shelterin mutations.

It is estimated that up to $30 \%$ of DKC cases do not have a known underlying genetic defect $(57,58)$. Thus, it is interesting to speculate whether new shelterin gene mutations might 
be discovered in human DKC. Based on our results, we predict that complete loss of shelterin function would not be compatible with life, as evidenced, for example, by in utero death upon complete loss of $A c d$ in fetal hematopoiesis. However, it is possible that relevant mutations decrease rather than abolish Acd expression. In mice, the hypomorphic acd splice variant was identified as a spontaneous mutation compatible with survival, but leading to a developmental syndrome with features of DKC, including profound HSC dysfunction. These observations suggest that mutations in gene regulatory regions or introns resulting in impaired gene expression should be considered when probing uncharacterized DKC cases. Furthermore, recent studies have identified a direct interaction between TERT and TPP1 that is critical for telomerase localization and processivity $(59,60)$. It is possible that specific mutations affecting this interaction could affect the ability of TPP1 to recruit telomerase without disrupting its function in preventing an uncontrolled DNA damage response, leading to a DKC-like phenotype. It has also been suggested that drugs targeting the TPP1-TERT interface could be used as anticancer agents to block telomere elongation in cancer cells; our observations indicate that it will be essential to ensure that these drugs do not interfere with other functions of TPP1 in order to avoid deleterious effects on the bone marrow. Finally, shelterin mutations may have to be considered in a broader range of human syndromes including those in patients with early-onset organ failure (as with TINF2 mutations) and with developmental disorders not previously associated with shelterin dysfunction.

A recent report has linked inactivation of the $\operatorname{Tr} f 1$ shelterin gene to an acute form of bone marrow failure in mice (61). These findings may be relevant to human disease, as TINF2 mutations in early-onset DKC cluster in a region encoding a TRF1-binding site. Interestingly, Trf1 inactivation led to rapid loss of hematopoietic progenitors and bone marrow failure, initially without detectable telomere shortening. When residual bone marrow cells were studied within 1 to 3 weeks after the induction of Cre recombinase, increased compensatory proliferation was apparent, leading eventually to telomere shortening and $p 21$ activation. These findings were proposed to underlie the pathogenic effects of shelterin mutations in human DKC through telomere deprotection, leading to rapid HSC loss. Our observations in Acd-deficient mice share key features with this report, including profound HSC depletion independent of telomere shortening. However, near-complete HSC depletion was apparent in our study already within 5 days after Acd inactivation, with a detectable DNA damage response and cell cycle arrest within 48 hours. To capture the direct cellular and molecular consequences of shelterin deprotection, we focused on very early time points before affected HSCs are depleted. This strategy identified increased telomeric fusion events and cell cycle arrest within 3 days of Acd inactivation. In contrast, Blasco and collaborators had to delay analysis or decrease the efficiency of target gene inactivation to preserve enough progenitors for study, which may have led to a strong selection for cells that escape Cre-mediated excision. In this situation, findings would focus on rare HSCs escaping inactivation and with functional shelterin that undergo major replicative stress, rather than on direct effects of shelterin deprotection. It is possible that these considerations may account for differences in our observations on Acd deficiency and those reported on the effects of $\operatorname{Tr} 1$ inactivation.
While it remains to be determined whether Acd mutations contribute to human bone marrow failure and DKC, mouse models of telomere deprotection through Acd mutation or deletion suggest this possibility. Furthermore, the hyperacute nature of hematopoietic defects following TPP1 loss provides a practical approach that could shed light on consequences of telomere crisis in other models of bone marrow failure. It is widely assumed that shortened telomeres induce a cellular crisis when their length becomes inadequate for the recruitment of an efficient shelterin buffer, unleashing a DNA damage response that can cause cell cycle arrest, apoptosis, or genetic instability. However, it is virtually impossible to study this pathogenic process directly in somatic stem cells of mice and patients, as telomere shortening occurs progressively and asynchronously in different stem cells over years in patients and over several generations in mouse DKC models. Furthermore, relevant stem cell populations are by definition profoundly depleted by the time of diagnosis in patients with bone marrow failure. In contrast, our study provides a well-defined acute window of observation on a cohort of hematopoietic progenitors that simultaneously and acutely undergoes telomere deprotection. Thus, our study presents new insights into early hematopoietic defects that may predate bone marrow failure in DKC patients and could be beneficial in developing a more complete understanding of the natural progression of human bone marrow failure syndromes.

\section{Methods}

Mice. C57BL/6 (B6, CD45.2+) mice were obtained from Harlan, and C57BL/6.Ptprca (B6-SJL, CD45.1 $1^{+}$) mice were obtained from the National Cancer Institute. acd hypomorphic mutant mice and mice carrying a floxed conditional $A c d$ allele $\left(A c d^{f l}\right)$ have been described $(8,30)$. $A c d^{f /+}$ mice were crossed with mice carrying a null $A c d$ allele $\left(A c d^{-}\right)$and $M x$-Cre (38), Vav-Cre transgenic (62), or Cre-ERT2 lentitransgenic mice (40). When indicated, mice were crossed onto a $p 53^{-/-}$background (63). To fully inactivate $A c d$, Cre-expressing $A c d^{l /-}$ mice were used. $M x$-Cre activation was initially achieved via five i.p. injections of $200 \mu \mathrm{g}$ poly(I:C) (EMD Millipore). To better capture acute changes induced by $A c d$ loss in hematopoietic stem and progenitor cells, we used a single $20-\mu$ g dose of highly purified poly(I:C) (GE Healthcare). This allowed the detection of hematopoietic progenitors soon after administration, with minimal effects on their phenotypic profile from the poly(I:C)-mediated interferon response. Cre-ERT2 activation was achieved by two to three i.p. injections of tamoxifen in corn oil (1 mg/dose; Sigma-Aldrich).

Flow cytometry. Fetal liver and bone marrow specimens were harvested, and single-cell suspensions were prepared. Red blood cells were lysed with ACK lysis buffer (Cambrex) and counted with a hemacytometer or a Nexcelom Auto T4 Cellometer (Nexcelom). The following antibodies were from BioLegend, eBiosciences, or BD Biosciences: anti-CD3, CD4, CD8, CD11b, CD11c, CD19, CD48, CD150, GR1/Ly-6G, B220, NK1.1, TCR $\beta$, TCR $\gamma \delta$, cKit, and Sca-1. We used the following antibody cocktail to exclude Lineage ${ }^{+}$cells: anti-CD11b, GR1, CD11c, B220, CD19, CD3, TCR $\beta$, TCR $\gamma \delta$, CD8, NK1.1, and TER119. BrdU analysis was performed using a BrdU-labeling kit (BD Biosciences). DilC1(5) and MitoTracker Green staining was performed using reagents from Invitrogen according to the manufacturer's protocols. For DNA content and aneuploidy analysis, cells were stained with the appropriate cell surface antibodies, fixed, and permeabilized according to the protocol provided for BrdU labeling (BD) and stained overnight with $0.1 \mathrm{mg} / \mathrm{ml}$ 4'6-diamidino-2-phenylindole (DAPI; Sigma-Aldrich). Analysis was performed on a FACSCanto flow cytometer and sorting on a FACSAria II/III cell sorter (BD Biosciences). Dead cells were excluded with DAPI. Files were analyzed using FlowJo software (TreeStar Inc.). 
Bone marrow and fetal liver cell transplantation. Six- to 8-week-old B6-CD $45.1^{+}$recipient mice were lethally irradiated ( 9 Gy, ${ }^{37} \mathrm{Cs}$ source). Four hours later, mice were transplanted with the indicated bone marrow or fetal liver cells via tail-vein injection. For competitive transplantation, we mixed equal numbers of competitor B6-CD45.1 bone marrow and tester B6-CD45.2 $2^{+}$bone marrow or fetal liver cells.

Complete blood counts. Blood was obtained through retro-orbital bleeding and transferred to EDTA-treated tubes. Complete blood counts were analyzed on the Advia 120 Hematology System (Siemens).

Quantitative real-time PCR. For gene expression analyses, greater than 5,000 Lin-Sca- ${ }^{\text {hi }} \mathrm{cKit}^{\text {hi }}$ (LSK) progenitors were sort purified directly into TRIzol (Invitrogen). After RNA extraction, cDNA was generated using the SuperScript III Synthesis Kit (Invitrogen). Relative gene expression was measured using TaqMan (Applied Biosystems) primer and probe sets. Reactions were carried out on a Mastercycler realplex system (Eppendorf). Relative expression was calculated after normalization with Hprt1 expression using the $\Delta \Delta C T$ method. For quantification of target gene excision, 10,000 LSK progenitors were sort purified into Direct Lysis Buffer (Viagen Biotech) with $1 \mu \mathrm{l}$ proteinase $\mathrm{K}$. Samples were incubated overnight, and proteinase $\mathrm{K}$ was inactivated at $85^{\circ} \mathrm{C}$ for 1 hour. To assess excision, we used a primer pair specific for the floxed Acd exon 7 (F: CTAACCCTGTCCCAGCTTCT; R: TACAAGGACCTTTCAGCACC) and an exon 2-specific primer pair (F: TACTCCAGGACTCGGAGACT; R: TCTGACACAAGTAACACGGC), amplifying a sequence outside the floxed region. Amplification was performed with SYBR Green (Thermo Fisher Scientific) before quantification of the exon 7/exon 2 signal on a Mastercycler realplex ( $\Delta \Delta C T$ method). For measurement of telomere length, DNA was extracted with Direct Lysis buffer (Viagen Biotech). Primers specific for telomeric DNA (F: CGGTTT GTTTGGGTTTGGGTTTGGGTTTGGGTTTGGGTT; R: GGCTTGCCTTACCCTTACCCTTACCСТTACССТTACCCT) and the reference gene m36b4 (F: ACTGGTCTAGGACCCGAGAAG; R: TCAATGGTGCCTCTGGAGATT) were used with SYBR Green amplification on a Mastercycler realplex system, as previously described $(64,65)$. The abundance of telomeric DNA normalized to $\mathrm{m} 36 \mathrm{~b} 4$ signal was calculated using the $\Delta \Delta \mathrm{CT}$ method.

Apoptosis and necrosis assays. For caspase- $3 / 7$ activation, $10^{4}$ bone marrow cells were suspended in $50 \mu \mathrm{l}$ DMEM plus $4 \%$ FBS and mixed with $50 \mu \mathrm{l}$ resuspended Caspase-Glo 3/7 buffer (Promega). Triplicate samples were incubated for 30 minutes in the dark and analyzed with an Enspire 2300 Multilabel reader (PerkinElmer). For HMGB1, serum was collected and frozen at $-80^{\circ} \mathrm{C}$ until analysis. Triplicate samples were then subjected to ELISA with an HMGB1 ELISA kit according to the manufacturer's protocol (IBL International). The reading was performed using a Spectramax M3 (Molecular Devices).

Metaphase preparation and analysis. Bone marrow cells were cultured for 12 hours in S-clone SF-O3 medium (Iwai North America) with IL-3 (9 ng/ml), IL-6 (5 ng/ml), and stem cell factor (100 ng/ml) (Peprotech) at $5 \% \mathrm{CO}_{2}$ and $37^{\circ} \mathrm{C}$. KaryoMAX Colcemid (Invitrogen) was added at
$0.2 \mu \mathrm{g} / \mathrm{ml}$ for 3 to 4 hours at $37^{\circ} \mathrm{C}$. Metaphase spreads were then prepared as described (66). Samples were stained with a PNA-TelG-FITC probe (Bio Synthesis) and DAPI. Metaphases were analyzed and images acquired using a Nikon E800 microscope equipped with an Olympus DP-71 digital camera. Images were scored in a blinded fashion.

GM-CFU and single-colony PCR. Twenty thousand bone marrow cells were plated per milliliter of Methocult GF M3534 (STEMCELL Technologies). Colonies were scored 7-10 days later. Individual colonies were removed as a plug with methylcellulose and washed in PBS before DNA extraction in $100 \mu \mathrm{l}$ lysis buffer (Viagen Biotech) with $1 \mu \mathrm{l}$ proteinase K. Samples were incubated overnight, and proteinase $\mathrm{K}$ was inactivated at $85^{\circ} \mathrm{C}$ for 1 hour. Colony DNA was then genotyped as described (8).

Statistics. Comparison of two means was performed with a 2-tailed unpaired Student $t$ test. Analysis of greater than two means was performed with ANOVA followed by Bonferroni's post-test analysis. Comparisons of observed genotypes with those predicted by Mendelian ratios were performed with $\chi^{2}$ analysis. $P<0.05$ was used as a threshold for statistical significance.

Study approval. All experiments in animals were approved by the University Committee on Use and Care of Animals (UCUCA) of the University of Michigan.

\section{Acknowledgments}

We thank Dr. Eric Brown (University of Pennsylvania, Philadelphia, Pennsylvania, USA) for the Cre-ERT2 lentitransgenic mice. This work was supported by the University of Michigan Biological Sciences Scholars Program (to I. Maillard) and the NIH (HL079118, to D.O. Ferguson; HD058606, to C.E. Keegan; and AI091627, to I. Maillard). Individual support included T32 training grants from the University of Michigan's Medical Scientist Training Program (GM07863, to M. Jones and J.A. Regal), the University of Michigan's Center for Organogenesis (HD007505, to M. Jones), and the University of Michigan's Cancer Biology Training Program (CA009676, to J.A. Regal). Flow cytometry was partially supported by a University of Michigan Cancer Center Grant (P30-CA46592).

Received for publication August 19, 2013, and accepted in revised form October 3, 2013.

Address correspondence to: Catherine E. Keegan, MSRB I \#3520, 1150 W. Medical Center Drive, University of Michigan, Ann Arbor, Michigan 48109, USA. Phone: 734.763.6767; Fax: 734.763.9512; E-mail: keeganc@med.umich.edu. Or to: Ivan Maillard, Life Sciences Institute \#6382A, 210 Washtenaw Avenue, University of Michigan, Ann Arbor, Michigan 48109, USA. Phone: 734.763.3599; Fax: 734.647.9702; E-mail: imaillar@umich.edu.
1. Greenberg RA, Allsopp RC, Chin L, Morin GB, Depinho RA. Expression of mouse telomerase reverse transcriptase during development, differentiation and proliferation. Oncogene. 1998;16(13):1723-1730.

2. Breault DT, et al. Generation of mTert-GFP mice as a model to identify and study tissue progenitor cells. Proc Natl Acad Sci U S A. 2008;105(30):10420-10425.

3. Lee HW, et al. Essential role of mouse telomerase in highly proliferative organs. Nature. 1998;392(6676):569-574.

4. Palm W, De Lange T. How shelterin protects mammalian telomeres. Annu Rev Genet. 2008;42:301-334.

5. Denchi EL, De Lange T. Protection of telomeres through independent control of ATM and ATR by TRF2 and POT1. Nature. 2007;448(7157):1068-1071.
6. Guo X, et al. Dysfunctional telomeres activate an ATM-ATR-dependent DNA damage response to suppress tumorigenesis. EMBO J. 2007; 26(22):4709-4719.

7. Hockemeyer D, et al. Telomere protection by mammalian Pot 1 requires interaction with Tpp1. Nat Struct Mol Biol. 2007;14(8):754-761.

8. Kibe T, Osawa GA, Keegan CE, De Lange T. Telomere protection by TPP 1 is mediated by POT1a and POT1b. Mol Cell Biol. 2010;30(4):1059-1066.

9. Wang F, et al. The POT1-TPP1 telomere complex is a telomerase processivity factor. Nature. 2007; 445(7127):506-510.

10. Xin $\mathrm{H}$, et al. TPP1 is a homologue of ciliate TEBP-beta and interacts with POT1 to recruit telomerase. Nature. 2007;445(7127):559-562.
11. Chong L, et al. A human telomeric protein. Science. 1995;270(5242):1663-1667.

12. Bilaud T, Brun C, Ancelin K, Koering CE, Laroche T, Gilson E. Telomeric localization of TRF2, a novel human telobox protein. Nat Genet. 1997; 17(2):236-239.

13. Broccoli D, Smogorzewska A, Chong L, De Lange T. Human telomeres contain two distinct Myb-related proteins, TRF1 and TRF2. Nat Genet. 1997; 17(2):231-235.

14. Smogorzewska A, De Lange T. Different telomere damage signaling pathways in human and mouse cells. EMBOJ. 2002;21(16):4338-4348.

15. Ye JZ, et al. TIN2 binds TRF1 and TRF2 simultaneously and stabilizes the TRF2 complex on telomeres. J Biol Chem. 2004;279(45):47264-47271. 
16. Takai KK, Kibe T, Donigian JR, Frescas D, De Lange $\mathrm{T}$. Telomere protection by TPP1/POT 1 requires tethering to TIN2. Mol Cell. 2011;44(4):647-659.

17. Pardo B, Marcand S. Rap1 prevents telomere fusions by nonhomologous end joining. EMBO J. 2005; 24(17):3117-3127.

18. Bae NS, Baumann P. A RAP1/TRF2 complex inhibits nonhomologous end-joining at human telomeric DNA ends. Mol Cell. 2007;26(3):323-334.

19. Sarthy J, Bae NS, Scrafford J, Baumann P. Human RAP1 inhibits non-homologous end joining at telomeres. EMBO J. 2009;28(21):3390-3399.

20. Hockemeyer D, Daniels JP, Takai H, De Lange $\mathrm{T}$. Recent expansion of the telomeric complex in rodents: Two distinct POT1 proteins protect mouse telomeres. Cell. 2006;126(1):63-77.

21. Palm W, Hockemeyer D, Kibe T, De Lange T. Functional dissection of human and mouse POT1 proteins. Mol Cell Biol. 2009;29(2):471-482.

22. $\mathrm{He} \mathrm{H}$, et al. Pot $1 \mathrm{~b}$ deletion and telomerase haploinsufficiency in mice initiate an ATR-dependent DNA damage response and elicit phenotypes resembling dyskeratosis congenita. Mol Cell Biol. 2009; 29(1):229-240.

23. Calado RT, Young NS. Telomere diseases. N Engl J Med. 2009;361(24):2353-2365.

24. Savage SA, Calado RT, Xin ZT, Ly H, Young NS, Chanock SJ. Genetic variation in telomeric repeat binding factors 1 and 2 in aplastic anemia. Exp Hematol. 2006;34(5):664-671.

25. Walne AJ, Vulliamy T, Beswick R, Kirwan M, Dokal I. TINF2 mutations result in very short telomeres: analysis of a large cohort of patients with dyskeratosis congenita and related bone marrow failure syndromes. Blood. 2008;112(9):3594-3600.

26. Savage SA, Giri N, Baerlocher GM, Orr N, Lansdorp PM, Alter BP. TINF2, a component of the shelterin telomere protection complex, is mutated in dyskeratosis congenita. Am J Hum Genet. 2008; 82(2):501-509.

27. Sarper N, Zengin E, Kilic SC. A child with severe form of dyskeratosis congenita and TINF2 mutation of shelterin complex. Pediatr Blood Cancer. 2010; 55(6):1185-1186

28. Vulliamy TJ, et al. Differences in disease severity but similar telomere lengths in genetic subgroups of patients with telomerase and shelterin mutations. PLoS One. 2011;6(9):e24383.

29. Vulliamy T, Beswick R, Kirwan MJ, Hossain U, Walne AJ, Dokal I. Telomere length measurement can distinguish pathogenic from non-pathogenic variants in the shelterin component, TIN2. Clin Genet. 2012;81(1):76-81.

30. Keegan CE, et al. Urogenital and caudal dysgenesis in adrenocortical dysplasia (acd) mice is caused by a splicing mutation in a novel telomeric regulator. Hum Mol Genet. 2005;14(1):113-123.

31. Tejera AM, et al. TPP1 is required for TERT recruitment, telomere elongation during nuclear reprogramming, and normal skin development in mice. Dev Cell. 2010;18(5):775-789.
32. Vlangos CN, O’Connor BC, Morley MJ, Krause AS, Osawa GA, Keegan CE. Caudal regression in adrenocortical dysplasia (acd) mice is caused by telomere dysfunction with subsequent p53-dependent apoptosis. Dev Biol. 2009;334(2):418-428.

33. Else T, et al. Genetic p53 deficiency partially rescues the adrenocortical dysplasia phenotype at the expense of increased tumorigenesis. Cancer Cell. 2009;15(6):465-476.

34. Wang Y, Shen MF, Chang S. Essential roles for Pot1b in HSC self-renewal and survival. Blood. 2011;118(23):6068-6077.

35. Else T, et al. Tpp1/Acd maintains genomic stability through a complex role in telomere protection. Chromosome Res. 2007;15(8):1001-1013.

36. Kiel MJ, Yilmaz OH, Iwashita T, Yilmaz OH, Terhorst C, Morrison SJ. SLAM family receptors distinguish hematopoietic stem and progenitor cells and reveal endothelial niches for stem cells. Cell. 2005; 121(7):1109-1121.

37. Kim I, He S, Yilmaz OH, Kiel MJ, Morrison SJ. Enhanced purification of fetal liver hematopoietic stem cells using SLAM family receptors. Blood. 2006; 108(2):737-744.

38. Kuhn R, Schwenk F, Aguet M, Rajewsky K. Inducible gene targeting in mice. Science. 1995 269(5229): 1427-1429.

39. Essers MA, et al. IFN $\alpha$ activates dormant haematopoietic stem cells in vivo. Nature. 2009; 458(7240):904-908.

40. Ruzankina Y, et al. Deletion of the developmentally essential gene ATR in adult mice leads to age-related phenotypes and stem cell loss. Cell Stem Cell. 2007; 1(1):113-126.

41. Chen MJ, Yokomizo T, Zeigler BM, Dzierzak E, Speck NA. Runx1 is required for the endothelial to haematopoietic cell transition but not thereafter. Nature. 2009;457(7231):887-891.

42. Stadtfeld M, Graf T. Assessing the role of hematopoietic plasticity for endothelial and hepatocyte development by non-invasive lineage tracing. Development. 2005;132(1):203-213.

43. Warr MR, et al. FOXO3A directs a protective autophagy program in haematopoietic stem cells. Nature. 2013;494(7437):323-327.

44. Scaffidi P, Misteli T, Bianchi ME. Release of chromatin protein HMGB1 by necrotic cells triggers inflammation. Nature. 2002;418(6894):191-195.

45. Sahin E, et al. Telomere dysfunction induces metabolic and mitochondrial compromise. Nature. 2011; 470(7334):359-365.

46. Chen LY, et al. Mitochondrial localization of telomeric protein TIN2 links telomere regulation to metabolic control. Mol Cell. 2012;47(6):839-850.

47. Castedo M, Perfettini JL, Roumier T, Andreau K, Medema R, Kroemer G. Cell death by mitotic catastrophe: a molecular definition. Oncogene. 2004; 23(16):2825-2837.

48. Castedo M, et al. Mitotic catastrophe constitutes a special case of apoptosis whose suppression entails aneuploidy. Oncogene. 2004;23(25):4362-4370.
49. Micheau O, Tschopp J. Induction of TNF receptor I-mediated apoptosis via two sequential signaling complexes. Cell. 2003;114(2):181-190.

50. Micheau $\mathrm{O}$, et al. The long form of FLIP is an activator of caspase- 8 at the Fas death-inducing signaling complex. J Biol Chem. 2002;277(47):45162-45171.

51. Tait SW, Green DR. Mitochondria and cell death: outer membrane permeabilization and beyond. Nat Rev Mol Cell Biol. 2010;11(9):621-632.

52. Thanasoula $\mathrm{M}$, et al. p53 prevents entry into mitosis with uncapped telomeres. Curr Biol. 2010; 20(6):521-526.

53. Cosme-Blanco W, et al. Telomere dysfunction suppresses spontaneous tumorigenesis in vivo by initiating p53-dependent cellular senescence. EMBO Rep. 2007;8(5):497-503.

54. D'adda Di Fagagna F, et al. A DNA damage checkpoint response in telomere-initiated senescence. Nature. 2003;426(6963):194-198.

55. Herbig U, Jobling WA, Chen BP, Chen DJ, Sedivy JM. Telomere shortening triggers senescence of human cells through a pathway involving ATM, p53, and p21(CIP1), but not p16(INK4a). Mol Cell. 2004; 14(4):501-513.

56. Hockemeyer D, Palm W, Wang RC, Couto SS, De Lange T. Engineered telomere degradation models dyskeratosis congenita. Genes Dev. 2008; 22(13):1773-1785.

57. Ballew BJ, Savage SA. Updates on the biology and management of dyskeratosis congenita and related telomere biology disorders. Expert Rev Hematol. 2013;6(3):327-337.

58. Savage SA, Alter BP. Dyskeratosis congenita. Hematol Oncol Clin North Am. 2009;23(2):215-231.

59. Nandakumar J, Bell CF, Weidenfeld I, Zaug AJ, Leinwand LA, Cech TR. The TEL patch of telomere protein TPP1 mediates telomerase recruitment and processivity. Nature. 2012;492(7428):285-289.

60. Zhong FL, Batista LF, Freund A, Pech MF, Venteicher AS, Artandi SE. TPP1 OB-fold domain controls telomere maintenance by recruiting telomerase to chromosome ends. Cell. 2012; 150(3):481-494

61. Beier F, Foronda M, Martinez P, Blasco MA. Conditional TRF1 knockout in the hematopoietic compartment leads to bone marrow failure and recapitulates clinical features of Dyskeratosis congenita. Blood. 2012;120(15):2990-3000.

62 . De Boer J, et al. Transgenic mice with hematopoietic and lymphoid specific expression of Cre. Eur J Immunol. 2003;33(2):314-325

63. Jacks T, et al. Tumor spectrum analysis in p53-mutant mice. Curr Biol. 1994;4(1):1-7.

64. Cawthon RM. Telomere measurement by quantitative PCR. Nucleic Acids Res. 2002;30(10):e47.

65. Callicott RJ, Womack JE. Real-time PCR assay for measurement of mouse telomeres. Comp Med. 2006; 56(1):17-22.

66. Lansdorp PM, et al. Heterogeneity in telomere length of human chromosomes. Hum Mol Genet. 1996;5(5):685-691. 